\title{
Lattice anisotropy as microscopic origin of static stripes in cuprates
}

\author{
B. Normand and A. P. Kampf \\ Theoretische Physik III, Elektronische Korrelationen und Magnetismus, Institut für Physik, \\ Universität Augsburg, D-86135 Augsburg, Germany.
}

(October 22, 2018)

\begin{abstract}
Structural distortions in cuprate materials offer a microscopic origin for anisotropies in electron transport in the basal plane. Using a real-space Hartree-Fock approach, we consider the ground states of the anisotropic Hubbard $\left(t_{x} \neq t_{y}\right)$ and $t-J\left(t_{x} \neq t_{y}, J_{x} \neq J_{y}\right)$ models. Symmetrical but inhomogeneous ("polaronic") charge structures in the isotropic models are altered even by rather small anisotropies to one-dimensional, stripe-like features. We find two distinct types of stripe, namely uniformly filled, antiphase domain walls and non-uniform, half-filled, in-phase ones. We characterize their properties, energies and dependence on the model parameters, including filling and anisotropy in $t$ (and $J$ ). We discuss the connections among these results, other theoretical studies and experimental observation.
\end{abstract}

\section{INTRODUCTION}

The issue of inhomogeneous charge and spin order in cuprate systems, or "stripe phases", has become one of the most controversial issues in the debate over hightemperature superconductivity. Experimentally, the evidence for static stripe phases in a variety of materials is incontrovertible: neutron diffraction studies of the Nd-doped LSCO system $\mathrm{La}_{2-x-y} \mathrm{Nd}_{y} \mathrm{Sr}_{x} \mathrm{CuO}_{4} \mathrm{H}^{3}$ show over the superconducting range of $x$ a charge modulation which has been interpreted as arising from a system of charged, alternating $(1,0)$ and $(0,1)$ domain walls separating antiferromagnetic (AF) regions where no holes are present. A similar phenomenon has been reported more recently in very low-doped LSCO without $\mathrm{Nd}$, although in this fase the stripes are diagonal and the system insulating 5.6 The variation of this inhomogeneous charge structure with hole doping $x$ has been characterized from the incommensurate magnetic diffraction peaks due to the antiphase nature of the domain walls.3. Although dynamical stripes (below) have been proposed as a pairing mechanism, also beyond dispute is that static stripe formation is inimical to superconductivity, as observed both from the individual, possibly coexisting, order parameters at fixed $\mathrm{Nd}$ contept $y, \mathrm{~B}$ and more broadly from the evolution of $T_{c}$ with $y$

Somewhat less unanimity is achieved concerning the existence and role of dynamical stripes, by which is meant charge fluctuations of the same incommensurate type. In contrast to early proposals based on the shape of the Fermi surface, 10 such fluctuations are now the favored interpretation of the incommensurate peaks pserved by inelastic neutron scattering in both LSCO11]12, 1 and YBCO13,14 systems. Nuclear Quadrupole Resonance (NQR) measurements15 17 suggest that charge-ordering features are ubiquitous, in materials both with and without Nd, on the short timescales probed by this technique. Indirect evidence for dynamical stripes has also been inferred from the suppression of $T_{c}$ on impurity doping, by the argument that pinning effects lead to static stripe formation (see Ref. 18, and references therein).

On the theoretical side, indications for stripe phases predate their experimental observation by some 6 years. Initial results from the real-space Hartree-Fock approach applied to a three-band Hubbard model 19 were followed by similar studies in a selection of systems, 2025 and by proposals based on frustrated phase separation.26 A dramatic increase in this activity since the discovery of stripes in nickelates and cuprates has seen charge inhomogeneities investigated further in Hubbard- and $t$ - $J$-based models by the Hartree-Fock (HF) approximation 27 $28 \mathrm{HF}$ with additional correjafions and static phonons, 29 the slave-boson technique, $30 \mathrm{nu}$ merical Densify-Matrix Renormalization Group (DMRG) simulatiops, 3133 the Dynamical Mean-Field Theory (DMFT), 34 and Exact Diagonalization (ED).33 The results of all of these studies imply that one-dimensional (1d) charge ordering is a leading instability in this class of models throughout the physical parameter regime Further analyses using the nonlinear sigma model, 35 coupled Luttinger liquids, 36 and Cluster Perturbation Theory (CPT) 37 adopt this striped state at the outset and shed additional light on its microscopic nature.

At the microscopic level, the relationship between structure and superconductivity has also been investigated since before the first reports of chargeinhomogeneous phases. Büchner et al.8 noted the structural transformation and loss of superconductivity on increasing $\mathrm{Nd}$ doping in a series of samples $\mathrm{La}_{2-x-y} \mathrm{Nd}_{y} \mathrm{Sr}_{x} \mathrm{CuO}_{4}$, and were further able to relate these results to the bond lengths and angle distortions in the low-temperature tetragonal (LTT) phase. Such analyses have been extended within the LSCO system to cover a variety of substituents 38 and also towards the use of dynamical probes to investigate the relation of structure and spin correlations (see for example Ref. 39). Of particular interest in connection with our analysis is the LTT structural transition also caused by Eu doping, and the $\mathrm{Cu}$ NQR studies 40 of this phase which indicate the presence of three inequivalent $\mathrm{Cu}$ sites depending on 
the location of the atom within or outside charged stripe structures. The microscopic relationship between lattice structure and the stripe instability has also been studied recently by detailed experiments focusing on bond lengths, or micro-strain, in the $\mathrm{CuO}$ polyhedra.41

In this work we begin with the experimental motivation provided - and quantified - by Ref. 8 , that real cuprate systems are often anisotropic, and that increase of this anisotropy supports static stripe formation. Theoretically, this suggests that appropriate starting models for the underlying physics of the cuprate planes would be anisotropic Hubbard $\left(t_{x} \neq t_{y}\right)$ or $t-J\left(t_{x} \neq t_{y}, J_{x} \neq J_{y}\right)$ models. 47 To investigate the relationship between this anisotropy and $1 \mathrm{~d}$, static charge order, we will adopt the most straightforward method known to provide initial, qualitative insight, and conduct real-space HF studies of anisotropic Hubbard and $t-J$ models. We aim to illustrate the effects of anisotropies on the static charge and spin configurations, and also to elucidate the energetic contributions (kinetic vs. magnetic) which drive the formation of inhomogeneous structures. The unrestricted HF technique is by nature suitable only for discussing the static properties, as it neglects quantum fluctuations which are essential for dynamical properties and restoration of broken symmetries. Thus we will not address the contentious issues of dynamical stripes or the connection to superconductivity. However, we hope to provide a suitable foundation on which to base further considerations, and will discuss below some more sophisticated approaches to the anisotropic models which may shed light on these questions, as well as on the roles of quantum fluctuations and lattice degrees of freedom which are neglected at this level.

The structure of the paper is as follows. In section II we review the experimental observations of structural anisotropies in cuprate materials. In Sec. III we give a brief account of the real-space Hartree-Fock method. Sec. IV presents results obtained for the Hubbard model with variable hopping anisotropy, and a comprehensive investigation of their evolution with the other intrinsic and extrinsic system parameters. In Sec. V we consider the $t-J$ model, provide analogous results where possible for anisotropies in both $t$ and $J$, and compare the two models. Sec. VI contains a discussion of the results in the context of experiment, some consideration of other materials, models and theoretical approaches, and a summary of our analysis.

\section{STRUCTURAL DISTORTIONS IN CUPRATES}

Before the first observations of stripes in any oxides, doping of LSCO by rare-earth elements was found to cause a structural transition from the low-temperature orthorhombic (LTO) phase to LTT.42 Büchner et al. investigated the suppression of superconductivity by Nd-doping in LSCO, 1 finding a phase diagram for
$\mathrm{La}_{2-x-y} \mathrm{Nd}_{y} \mathrm{Sr}_{x} \mathrm{CuO}_{4}$ with the LTO phase (Fig. 1(a)) for $y<0.16$, LTT (Fig. 1(b)) with a superconducting ground state in the doping range $0.16<y<5 x-0.55$, and LTT but non-superconducting for $y>5 x-0.55$. For large $x$ the high-temperature tetragonal (HTT) phase remains stable down to low temperature, and for small $x$ with $y>0.16$, the structure is Pccn. By combining transport measurements with structural refinement, the line $y \simeq 5 x-0.55$ was found to correspond to a critical buckling angle of the $\mathrm{CuO}_{6}$ octahedra in the LTT structure, $\Phi_{c} \simeq 4^{\circ}$. A further key point in support of the close connection between charge order and lattice structure is the observation that the orientation of the stripes alternates between $(1,0)$ and $(0,1)$ on proceeding along the $\hat{c}$-axis, as does the rotation axis of the LTT structural distortion (Fig. 1(b)). It is clear that the electronic structure of the $\mathrm{CuO}_{2}$ plane is altared strongly by small changes in the lattice structure. 44

The LTO and LTT phases both involve a distortion of the flat $\mathrm{CuO}_{2}$ planes by rotation of the $\mathrm{CuO}_{6}$ structural units. For LTO this rotation takes place around a $(1,1)$ diagonal of the $\mathrm{CuO}_{2}$ system, such that all the inplane $\mathrm{O}$ atoms of the HTT structure are displaced from the plane. For LTT this rotation is around a $(0,1)$ or $(1,0)$ axis, such that only $x$-axis (or $y$-axis) $\mathrm{O}$ atoms are displaced. Structural analysis indicates that the fundamental $\mathrm{Cu}-\mathrm{O}$ separation is not altered significantly by the distortion, which is taken up rather by the $\mathrm{Cu}-\mathrm{O}-\mathrm{Cu}$ bond angle, 2థ. Inspection of Fig. 1 shows that (despite the crystallographic terminology, which is based on a unit cell of side $\sqrt{2}$ larger than the $\mathrm{Cu}-\mathrm{Cu}$ distance), this planar buckling affects the $\mathrm{Cu}-\mathrm{O}-\mathrm{Cu}$ bonds equally in $x$ - and $y$-directions in the LTO phase, but unequally in LTT.

This result provides a microscopic origin for in-plane anisotropies in the LSCO system. Calculation from first principles of the electron transfer and superexchange parameters, $t$ and $J$, indicate that in cuprate materials these deviate rather strongly 45 from the elementary orbital-overlap theory 4 For a $(1,0)$ LTT anisotropy, one expects an angular dependence given approximately by 47

$$
t_{y}=t_{x}|\cos (\pi-2 \Phi)|, \quad J_{y}=J_{x} \cos ^{2}(\pi-2 \Phi) .
$$

For a maximal distortion angle $\Phi=5^{\circ}$, the relative anisotropies $\left|t_{x}-t_{y}\right| / t_{x} \sim 1.5 \%$ and $\left|J_{x}-J_{y}\right| / J_{x} \sim 3 \%$ may appear rather small, but we note that even these small values result in the absolute differences $\left|t_{x}-t_{y}\right|$ and $\left|J_{x}-J_{y}\right|$ exceeding the superconducting $T_{c}$. The qualitative picture thus emerges of a possible microscopic origin for the formation of $1 \mathrm{~d}$ structures (stripes) as the true ground state of the weakly anisotropic electronic system, and it is this possibility which we wish to investigate. Competition of such a static stripe state with a superconducting one, which may be the true ground state of the $2 \mathrm{~d}$ system, would be fully consistent with experimental observation. We note in this connegtion that the issug of bulk coexistence of these two statest remains open. 18 

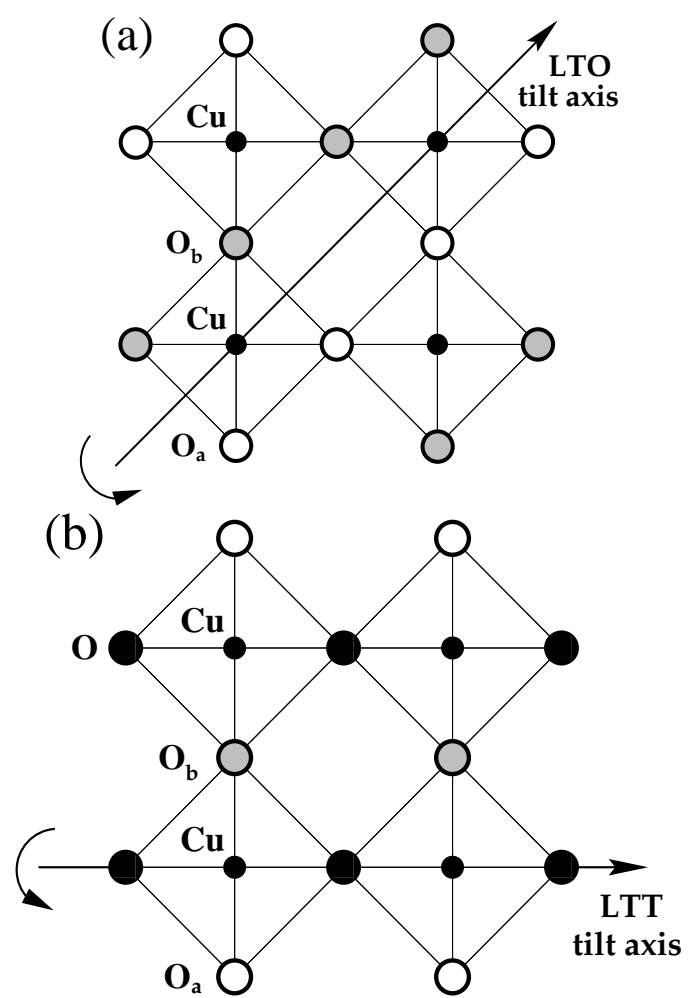

FIG. 1. Schematic representations of the conformation of the $\mathrm{CuO}_{2}$ plane in LTO (a) and LTT (b) lattice structures. Small, black circles represent $\mathrm{Cu}$ atoms, which remain in the plane in both structures. Large, black circles represent $O$ atoms which remain in the plane, white circles $\mathrm{O}$ atoms which are driven above the plane, and grey circles $\mathrm{O}$ atoms which are driven below it by the tilting distortion.

An important feature of the Nd-doped LSCO system which we have discussed is that the LTO-LTT transition and the formation of static stripes, or charge order, appear to be decoupled. For $y=0.4$ and in the optimal doping range of $x$, the structural transition occurs at a temperature $T_{\mathrm{LT}}, 80 \mathrm{~K}$, while charge order appears at some $T_{\mathrm{ch}}<40 \mathrm{~K}$. 117 Although these temperature scales become closer for some smaller values of $y$ and $x$, they show opposing trends as functions of $x$, and itappears justified to treat the two phenomena separately. 49 We will thus consider only the energetics of the electronic subsystem, studied on a rigidly distorted lattice. This is clearly a simplifying feature in comparison with, for example, highly-doped nickelate systems, where stripe formation and structural distortion appear to occur together as a single, first-order transition, and any energetic considerations would require a comparison between the gain in the electronic subsystem with the cost to the lattice.

In closing this section, we note that another manifestly anisotropic cuprate system is YBCO, where the $\hat{b}$-axis chains in the $\mathrm{Cu}(1)-\mathrm{O}$ layer cause a significant difference in the structural parameters of the $\mathrm{CuO}_{2}$ plane. The absence of static stripes in YBCO suggests that $x$ - and $y$-axis transfer integrals may in fact be rather similar (to within some critical ratio), or that the bilayer nature may be important, or may reflect the fact that the resultant stripe structure would be the same in every plane, possibly suffering additional penalties in Coulomb energy compared to the case of LTT LSCO, where the rotation axis and stripe direction alternate along the $\hat{c}$-axis.

\section{REAL-SPACE HARTREE-FOCK APPROXIMATION}

This technique has been employed by many authors to gain insight into the qualitative physics of the Hubbard model, and we provide only a brief overview of the steps involved in the procedure After the initial results of Zaanen and Gunnarsson, 19 who found stable, open and closed domain walls in a three-band Hubbard model, subsequent analyses 20 22, 44 of Hubbard models have verified this property in the one-band case, considered the evolution of stripe features with the ratio $U / t$ and with filling, and clarified both the filling of domain walls and the phase relationship between magnetic regions.27 Further refinements of the method have involved the inclusion of local correlations and static phonon terms. 29

The one-band Hubbard model is written as

$$
H=\sum_{i, \pm \eta, \sigma} t_{\eta}\left(c_{i \pm \eta \sigma}^{\dagger} c_{i \sigma}+H . c .\right)+U \sum_{i} n_{i \uparrow} n_{i \downarrow},
$$

where $n_{i \sigma}=c_{i \sigma}^{\dagger} c_{i \sigma}, \eta$ denotes $x, y$, and on an anisotropic lattice $t_{x} \neq t_{y}$. Denoting the terms in Eq. (2) by $H_{t}$ and $H_{U}$, in the HF approximation

$$
\begin{aligned}
H_{U}= & U \sum_{i}\left[\left\langle n_{i \uparrow}\right\rangle n_{i \downarrow}+n_{i \uparrow}\left\langle n_{i \downarrow}\right\rangle-\left\langle n_{i \uparrow}\right\rangle\left\langle n_{i \downarrow}\right\rangle\right. \\
& \left.-\left\langle c_{i \downarrow}^{\dagger} c_{i \uparrow}\right\rangle c_{i \uparrow}^{\dagger} c_{i \downarrow}-\left\langle c_{i \uparrow}^{\dagger} c_{i \downarrow}\right\rangle c_{i \downarrow}^{\dagger} c_{i \uparrow}+\left\langle c_{i \uparrow}^{\dagger} c_{i \downarrow}\right\rangle\left\langle c_{i \downarrow}^{\dagger} c_{i \uparrow}\right\rangle\right],
\end{aligned}
$$

where the signs include fermion statistics, precluding the need to form Slater determinants of quasiparticle states. We will focus here on collinear spin configurations, and not in spiral or vortex solutions.24 In this case, by choosing the axis-ff the possibly broken spin symmetry to be the $\hat{z}$-axis, 20 one may work without loss of generality using only the first line of Eq. (3).

The model is solved in real space on a small cluster of sites $i$. The particle number $q_{i}$ and magnetization components $m_{i}^{\alpha}$ are defined by the expectation values

$$
\begin{aligned}
q_{i} & =\left\langle c_{i \uparrow}^{\dagger} c_{i \uparrow}+c_{i \downarrow}^{\dagger} c_{i \downarrow}\right\rangle \\
m_{i}^{z} & =\frac{1}{2}\left\langle c_{i \uparrow}^{\dagger} c_{i \uparrow}-c_{i \downarrow}^{\dagger} c_{i \downarrow}\right\rangle \\
m_{i}^{x} & =\frac{1}{2}\left\langle c_{i \uparrow}^{\dagger} c_{i \downarrow}+c_{i \downarrow}^{\dagger} c_{i \uparrow}\right\rangle \\
m_{i}^{y} & =-\frac{1}{2} i\left\langle c_{i \uparrow}^{\dagger} c_{i \downarrow}-c_{i \downarrow}^{\dagger} c_{i \uparrow}\right\rangle .
\end{aligned}
$$

Solution proceeds by iteration of the site parameters to self-consistency, at which point one has solved all of the mean-field equations contained in Eq. (四), subject to the 


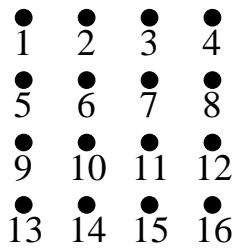

FIG. 2. Numbering scheme for an $l=n \times n$ cluster, illustrated with $n=4$.

additional constraint on the total particle number, which is implemented in the number of filled quasiparticle energy levels. This procedure is not identical to finding the global minimum of the multi-parameter free-energy surface, in that in principle it may only find stationary points, and the user is generally offered no guarantee that these correspond to global minima.

To solve the self-consistency problem for an $l=n \times n$ cluster requires diagonalization of the $2 n^{2} \times 2 n^{2}$ Hamiltonian matrix form of Eq. (2),

$$
H_{i j}=\left[\begin{array}{cccccc}
\mathbf{N}_{1} & \mathbf{M}_{12} & 0 & \mathbf{M}_{41}^{*} & \mathbf{M}_{15} & \ldots \\
\mathbf{M}_{12}^{*} & \mathbf{N}_{2} & \mathbf{M}_{23} & 0 & 0 & \ldots \\
0 & \mathbf{M}_{23}^{*} & \mathbf{N}_{3} & \mathbf{M}_{34} & 0 & \ldots \\
\vdots & \vdots & \vdots & \vdots & \vdots & \ddots
\end{array}\right]
$$

Here we have discarded the constant terms in Eq. (3), although these are restored in calculating the energy of the self-consistent solutions which emerge. The $2 \times 2$ "diagonal" and "off-diagonal" matrix elements are respectively

$$
\mathbf{N}_{i}=\left(\begin{array}{cc}
\frac{1}{2} U\left(q_{i}-2 m_{i}^{z}\right) & -U\left(m_{i}^{x}-i m_{i}^{y}\right) \\
-U\left(m_{i}^{x}+i m_{i}^{y}\right) & \frac{1}{2} U\left(q_{i}+2 m_{i}^{z}\right)
\end{array}\right)
$$

and

$$
\mathbf{M}_{i j}=\left(\begin{array}{cc}
t_{\eta} & 0 \\
0 & t_{\eta}
\end{array}\right)
$$

in which $\eta=x, y$ for $j=i+1(-n), i+n(-l)$, and the two-component structure corresponds to the two spin directions in the $S_{z}$ basis. For the numbering scheme illustrated in Fig. 2 with a $4 \times 4$ cluster, the off-diagonal blocks connect $i$ to $i \pm 1$ in the $\hat{x}$-direction, and to $i \pm n$ in the $\hat{y}$-direction. Open boundary conditions (BCs) are implemented with no forward (backward) connections in $x$ when $\bmod (i, n)=0(\bmod (i, n)=n-1)$, and in $y$ when $i>n^{2}-n(i \leq n)$. Periodic BCs have forward (backward) connections from $i$ to $i+1-n(i-1+n)$ in $x$, such as the element $\mathbf{M}_{41}$ in Eq. (5), and to $i+n-n^{2}$ $\left(i-n+n^{2}\right)$ in $y$ for the same edge sites. Because the Hamiltonian matrix is Hermitian, only half of these connections must be set explicitly. For cluster filling $x$, the lowest $2 n^{2} x$ eigenstates of the diagonal Hamiltonian matrix are filled, $q_{i}$ and $\mathbf{m}_{i}$ for all sites $i$ are deduced from the eigenvectors representing the new quasiparticles, the new matrix is diagonalized, and the process continued. The consistency condition was generally taken to have been achieved when the summed squares of the changes in $q_{i}$ and $m_{i}^{\alpha}$ were less than $10^{-8}$ per site, although this was continued in some cases to the machine precision to ensure that no further changes were possible. Convergence was usually achieved for $12 \times 12$ systems within 200 iterations using open $\mathrm{BCs}$, and within 1000 iterations for periodic BCs, where the iteration procedure could more clearly be observed converging on and diverging from a sequence of local minima.

The $t-J$ model has previously been considered in the real-space HF formalism only in Ref. 25. Here we have chosen to study the form

$$
H_{t-J}=H_{t}+H_{U}+J\left(\mathbf{S}_{i} \cdot \mathbf{S}_{j}-\frac{1}{4} n_{i} n_{j}\right),
$$

with $n_{i}=n_{i \uparrow}+n_{i \downarrow}$, and $U$ taken to have a large, finite value to mimic the projection property of the $t-J$ model onto only those states with no doubly occupied sites. We will have more to say on this approximation in Sec. V. In this case the $J$ term admits further decouplings, among which we neglect pairing terms when working in a canonical ensemble, and nearest-neighbor spin-flip terms because these are generally small. Thus we retain only a finite expectation value of the bond hopping term $A_{i j \sigma}=\left\langle c_{i \sigma}^{\dagger} c_{j \sigma}\right\rangle .25$

The HF decomposition of the additional $J$ term is

$$
\begin{aligned}
H_{J}=-\frac{1}{2} J & \sum_{\langle i j\rangle}\left[\left\langle n_{i \uparrow}\right\rangle n_{j \downarrow}+\left\langle n_{j \downarrow}\right\rangle n_{i \uparrow}-\left\langle n_{i \uparrow}\right\rangle\left\langle n_{j \downarrow}\right\rangle\right. \\
& +\left\langle n_{i \downarrow}\right\rangle n_{j \uparrow}+\left\langle n_{j \uparrow}\right\rangle n_{i \downarrow}-\left\langle n_{i \downarrow}\right\rangle\left\langle n_{j \uparrow}\right\rangle \\
& +A_{i j \uparrow} c_{j \downarrow}^{\dagger} c_{i \uparrow}+A_{i j \uparrow}^{*} c_{i \downarrow}^{\dagger} c_{j \uparrow}+A_{i j \uparrow}^{*} A_{i j \downarrow} \\
& \left.+A_{i j \uparrow} c_{j \downarrow}^{\dagger} c_{i \uparrow}+A_{i j \uparrow}^{*} c_{i \downarrow}^{\dagger} c_{j \uparrow}+A_{i j \uparrow}^{*} A_{i j \downarrow}\right] .
\end{aligned}
$$

The $2 n^{2} \times 2 n^{2}$ matrix to be diagonalized (cf. (5)) has

$$
\mathbf{N}_{i}=\left(\begin{array}{cc}
\frac{1}{2}\left[U\left(q_{i}-2 m_{i}^{z}\right)-\tilde{J}_{i \downarrow}\right] & -U\left(m_{i}^{x}-i m_{i}^{y}\right) \\
-U\left(m_{i}^{x}+i m_{i}^{y}\right) & \frac{1}{2}\left[U\left(q_{i}+2 m_{i}^{z}\right)-\tilde{J}_{i \uparrow}\right]
\end{array}\right)
$$

as the $2 \times 2$ diagonal component, where

$$
\tilde{J}_{\sigma}=J_{x}\left(n_{i+x \sigma}-n_{i-x \sigma}\right)+J_{y}\left(n_{i+y \sigma}-n_{i-y \sigma}\right),
$$

and

$$
\mathbf{M}_{i j}=\left(\begin{array}{cc}
t_{\eta}-\frac{1}{2} J_{\eta} A_{\eta \downarrow}^{*} & 0 \\
0 & t_{\eta}-\frac{1}{2} J_{\eta} A_{\eta \uparrow}^{*}
\end{array}\right) .
$$

in the non-zero, off-diagonal blocks of the upper triangle. Further details of the iterative calculations in this case are deferred to Sec. V.

\section{HUBBARD MODEL}

In this section we describe the charge and spin configurations (4) which emerge as the self-consistent solutions 
to the Hubbard model (2) in the Hartree-Fock approximation (3). We begin by considering the isotropic system as a function of the "intrinsic" system parameters, the ratio $U / t$, the (hole) filling $x$, and the temperature $T$, and make contact with the results of previous studies. We then illustrate how these results are altered in the presence of hopping anisotropy $t_{x} \neq t_{y}$, for which we study both the charge and spin distribution and the kinetic and magnetic energy components. We comment also on the variation of the solutions with "extrinsic" parameters, by which is meant cluster size, BCs, and commensuration. We now set the energy unit to be $t=1$. In all figures to follow the $\hat{x}$-axis is horizontal and the $\hat{y}$-axis vertical.

\section{A. Variation of $U$}

For small values of $U$ the solutions are homogeneous in charge and have no local magnetization. This "metallic" phase is the ground state for $U \lesssim 3$, the exact value of $U_{c}$ depending on the filling. We have not analyzed this regime or the metal-insulator transitiop in any detail, as many more accurate techniques exist. 50 For all values $U \gtrsim 3$, the predominant HF solutions are inhomogeneous charge clusters, with locally AF spin orientation. In the approximate range $3 \lesssim U \lesssim 6$, these clusters take the form of closed loops of electron-depleted sites, or domain walls enclosing an undoped region (Figs. 3(a) and 4(a)). We will call these formations "corrals", to distinguish them from the situation as $U$ is increased. For $U \gtrsim 6$ the charge clusters are better described as a solid region of hole doping, which we will call a "polaron" (Fig. 3(b)). Our results are rather similar to those in Fig. 1 of Vergés et al.24 for this isotropic situation.

\section{B. Variation of $x$}

As the filling is varied in the isotropic model we note the same general features, but with important alterations. With smaller hole doping the cluster is less influenced by the system boundaries, and prefers (Fig. 4(a)) "diagonal" corrals composed of $(1,1)$ domain walls, rather than the "vertical" type with $(1,0)$ domain walls (Fig. 3(a)). This result is quite ubiquitous, and readily understood from the kinetic energy gain due to nearest-neighbor hopping processes on all four bonds around one hole. Furthermore, applying periodic rather than open BCs leads to a division of large corrals into small, diagonal ones, which appear to form a lattice (Figs. 4(b), 5(a)). We may conclude that the vertical corral shown in Fig. 3(a) is constrained by the system dimensions, an observation confirmed at still higher filling.
Open BCs

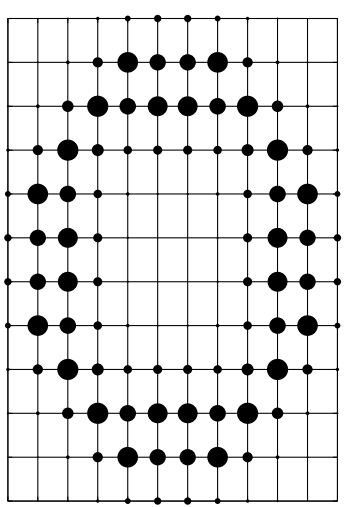

(a)

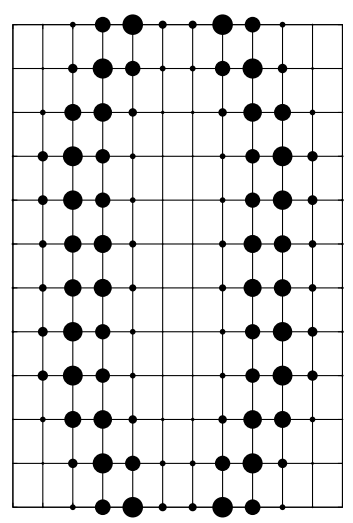

(c)

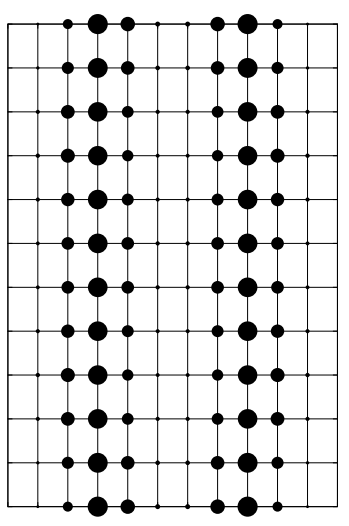

(e)
Open BCs

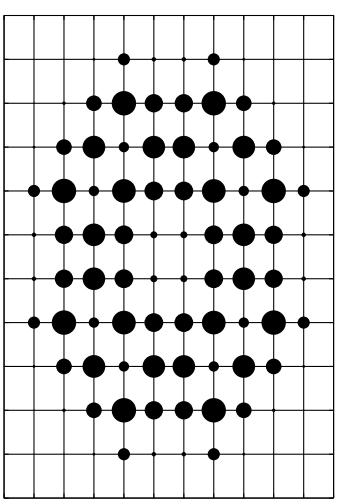

(b)

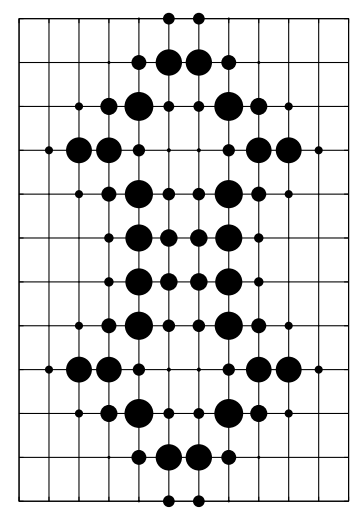

(d)

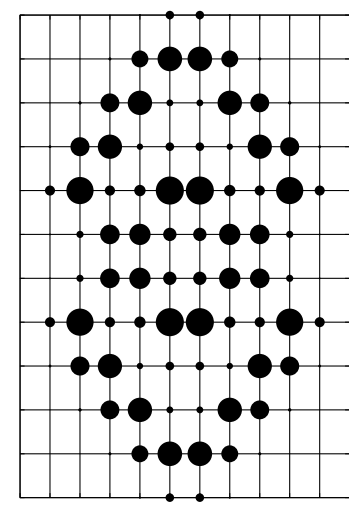

(f)
FIG. 3. Ground-state charge distributions for Hubbard model with hole doping $x=1 / 6$ on a $12 \times 12$ cluster with open BCs. (a) $U=5, t_{x}=t_{y}=-1$. (b) $U=8, t_{x}=t_{y}=-1$. (c) $U=5, t_{x}=-1.05, t_{y}=-0.95$. (d) $U=8, t_{x}=-1.05$, $t_{y}=-0.95$. (e) $U=5, t_{x}=-1.1, t_{y}=-0.9$. (f) $U=8$, $t_{x}=-1.1, t_{y}=-0.9$. In these figures the largest circles correspond to $\left\langle n_{i}\right\rangle=0.48$, or $52 \%$ hole doping of the site. 
Open BCs

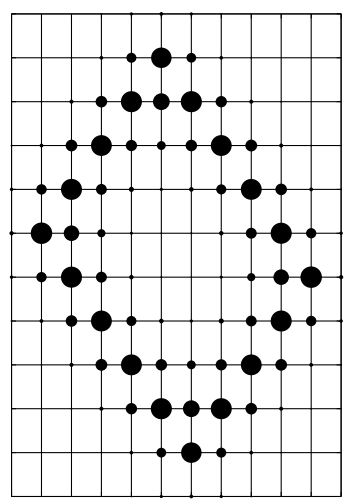

(a)

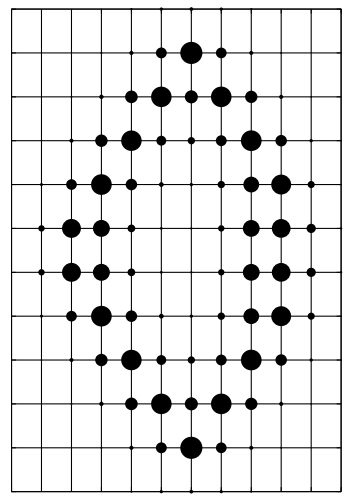

(c)

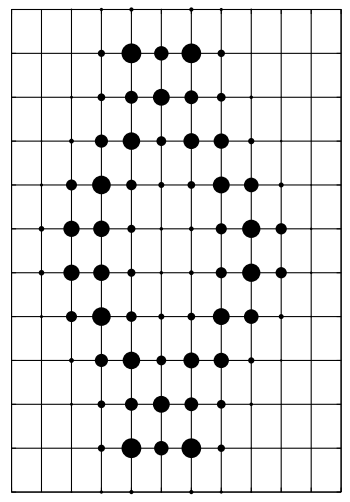

(e)
Periodic BCs

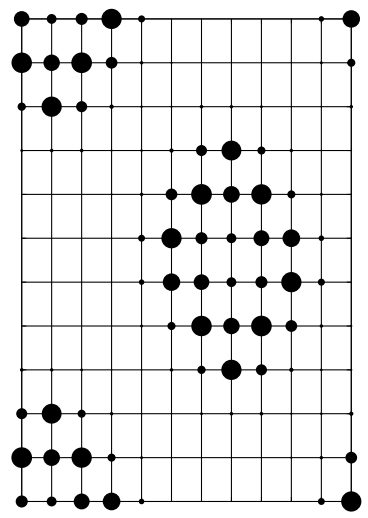

(b)

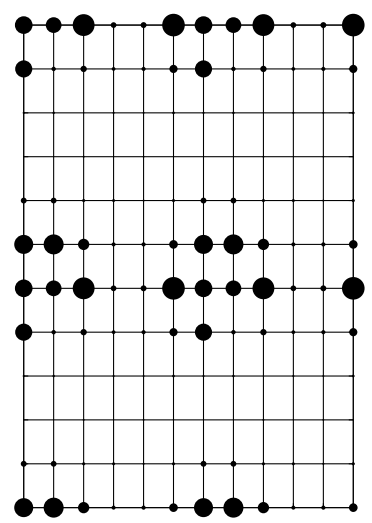

(d)

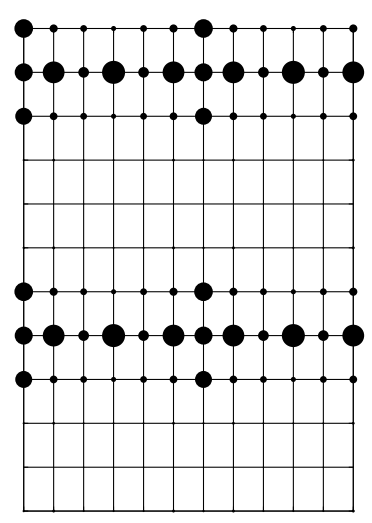

(f)

FIG. 4. Ground-state charge distribution for Hubbard model with hole doping $x=1 / 8$ and $U=5$ on a $12 \times 12$ cluster. (a) Open BCs, $t_{x}=t_{y}=-1$. (b) Periodic BCs, $t_{x}=t_{y}=-1$. (c) Open BCs, $t_{x}=-1.05, t_{y}=-0.95$. (d) Periodic BCs, $t_{x}=-1.05, t_{y}=-0.95$. (e) Open BCs, $t_{x}=-1.1, t_{y}=-0.9$. (f) Periodic BCs, $t_{x}=-1.1, t_{y}=-0.9$. Charge scale as in Fig. 3.

\section{Variation of temperature}

We have in addition considered the real-space HF approximation at finite temperature. This part of the anal-

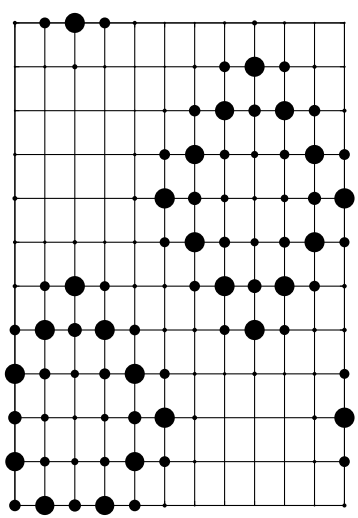

(a)

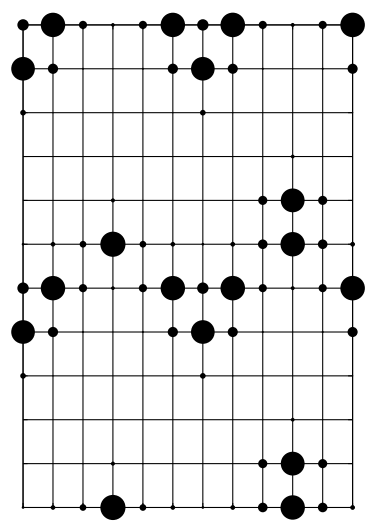

(b)
FIG. 5. Ground-state charge distribution for Hubbard models on a $12 \times 12$ cluster. (a) $U=5, x=1 / 6$, periodic BCs, $t_{x}=t_{y}=-1$. (b) $U=8, x=1 / 8$, periodic BCs, $t_{x}=-1.1, t_{y}=-0.9$. Charge scale as in Fig. 3 .

ysis was motivated by the picture of the self-consistency procedure as the following of a path through a free-energy "landscape". Because the solution is a stationary point, and not a global minimum, one would wish to sample as many local minima as possible, which is facilitated when the landscape be as smooth as possible. On descending from high temperatures, one expects a uniform charge distribution to be the state of minimum free energy, and that the leading instability to an inhomogeneous distribution would appear as a single minimum before the landscape could become more complex at lower temperatures. The effect of a finite temperature is readily incorporated by a thermal factor in each of the mean-field equations (4), and by replacing the condition on the number of quasiparticle states filled with an equation determining the Fermi energy.

At high temperatures, $T>2$, the solutions are always uniform in charge and have no magnetization. Depending on the exact choice of the other parameters, for temperatures in the range $0.18<T<2$, an AF spin configuration develops while the charge distribution remains uniform. Finally, at temperatures $T \sim 0.18$ for most of the situations we have considered, an inhomogeneous charge structure develops (corrals, polarons, stripes with suitable anisotropy) while the spins remain locally AF. In all the cases we considered, the leading instability to charge inhomogeneity at finite temperature was identical to the ground-state structure found by working entirely at $T=0$, with a single exception mentioned below. This result is reassuring in the sense of helping to confirm that the minima emerging from the zero-temperature studies are quite likely to be global in nature, and in this work we will not pursue the finite-temperature studies beyond establishing this point. We note finally that in this approach the onset temperature for formation of an incommensurate spin structure where this arises (e.g. Fig. 3(e)) 
is identical to that for charge order.

\section{Variation of anisotropy}

We turn now to the most important aspect of our analysis. Figs. 3 and 4 (c)-(f) illustrate the effects of increasing the hopping anisotropy $\epsilon_{t}=t_{x} / t_{y}-1$ to $11 \%$ and $22 \%$ for a selection of initial parameters and BCs. Figs. 3(a), (c) and (e) give the clearest demonstration of stripe formation as a result of hopping anisotropy; although this is for open BCs the results for periodic BCs are almost identical for this choice of $U$ and $x$. Figs. 3(b), (d) and (f) show that for stronger $U$ the polaronic state is more favorable, and although it becomes progressively more elongated with increasing anisotropy, stripe formation is not achieved at $22 \%$. Similarly, Figs. 4(a), (c) and (e) show the tendency of corrals to become increasingly diamond-shaped with anisotropy, before crossing to a fully $1 \mathrm{~d}$ state. The difference between these and the analogous cases in Fig. 3 is that for the smaller filling the (diagonal) corrals are rather more stable, and a greater anisotropy would be required to create the stripe state.

One of our key qualitative results is evident in these figures. The direction of the stripes, and the major axis of the diamonds, is perpendicular to the direction of the strong hopping. Stripes are stabilized not by charge motion along them (the picture of a conducting channel), but by transverse hopping,27.36 onto and off the stripe. This is readily shown by a simple argument based on site charge densities, which we illustrate for the 4-site cluster with two different densities $n_{1}$ and $n_{2}$ of Fig. 6 . For both spin species $\left(n_{\uparrow}, n_{\downarrow} \equiv n\right)$, a hopping amplitude proportional to the occupation of the initial state and the availability of the empty state gives

$$
\begin{aligned}
\left\langle E_{x}^{t}\right\rangle / t & =n_{1}\left(1-n_{1}\right)+n_{2}\left(1-n_{2}\right) \\
& =2\left(n_{1}+n_{2}-\left(n_{1}^{2}+n_{2}^{2}\right)\right) \\
\left\langle E_{y}^{t}\right\rangle / t & =n_{1}\left(1-n_{2}\right)+n_{2}\left(1-n_{1}\right) \\
& =2\left(n_{1}+n_{2}-2 n_{1} n_{2}\right),
\end{aligned}
$$

whence the identity $n_{1}^{2}+n_{2}^{2} \geq 2 n_{1} n_{2}$ ensures that $\left\langle E_{y}^{t}\right\rangle \geq$ $\left\langle E_{x}^{t}\right\rangle$. That hopping between two different charge densities is favored over that between equal densities ensures that transverse charge fluctuations determine the orientation of uniform stripe states such as those in Fig. 3(e).

This result should not be confused with the metallic or insulating nature of the stripes: these are still separated in the transverse direction by insulating, AF regions. Of all the charge-inhomogeneous solutions illustrated here, only the stripe solutions are metallic, and this in the stripe direction within the $2 \mathrm{~d}$ structure. While we have considered transverse hopping as uncorrelated, virtual processes, other microscopic approaches suggest a coherent hopping of stripe segments of lengths around 5 lattice sites 51

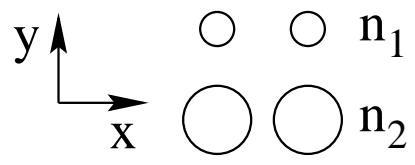

FIG. 6. Four-site cluster with two separate charge densities $n_{1}$ and $n_{2}$, to illustrate kinetic energy difference between $\hat{x}$ - and $\hat{y}$-directions (Eq. (13)).

The above reasoning clarifies the somewhat unexpected result in Figs. 4(d), 4(f) and 5(b): here the stripes are aligned in the direction of the stronger hopping. In this case the hole count at $x=1 / 8$ is insufficient for uniform stripe formation on a $12 \times 12$ cluster, and instead we find a non-uniform charge configuration. Such alternating stripes were presented in Fig. 1(c) of Ref. 29 (to be contrasted with the variant in Fig. 1(a) of the same work), and are one HF analogue of "half-filled" stripes.27 We will follow this terminology, but note in passing that the idealized stripe with half of its sites occupied by holes is in fact quarter-filled with electrons. The fact that hopping along the stripe proceeds between sites of high and low charge density helps to explain (13) the orientation of this type of $1 \mathrm{~d}$ charge inhomogeneity.

An enduring problem for HF studies of the Hubbard model has been that these show a definite preference for uniform, filled stripes, as in Fig. 3(e) where the hole density per site along the stripe is unity. By contrast, the experimental situation fayrers half-filled stripes, in the intermediate doping range. 1 . While there is to date no information to distinguish between the possibilities of uniform or alternating (Fig. 4(b)) hole distribution within such a stripe, one may expect thatter to be favored by increasing $U$. Some analytica 2251 and numerical 31 studies have addressed the issue of the additional physics which may be responsible for this result. Here we have found that hopping anisotropy represents an additional factor which may contribute to the stability of non-uniform, half-filled stripes. Although this phase is obtained at the "magic" filling $x=1 / 8$, close to which charge-ordering effects in experiment are strongest 17 we hesitate to comment on a connection due to the issue of commensuration effects on the finite cluster.

In common with other HF studies, this state is somewhat more fragile than the uniform stripe, as indicated by the fact that it is preferred over a corral only in the presence of periodic BCs, and has a smaller energy gain per hole than the uniform stripe (Table III below). This case (Fig. 4(f)) was the only situation in which the leading instability at high temperatures (a corral) was different from the low- $T$ structure. However, our result remains indicative of the possibilities allowed in the presence of hopping anisotropies, and we believe representative of the situation for appropriate fillings. At larger values of $U$ one observes the emergence of the same type of state, but also (Fig. 5(b)) that competition is more severe from a phase of isolated, small polarons (Fig. 1(d), Ref. 29). Increasing anisotropy promotes alignment of these polarons 


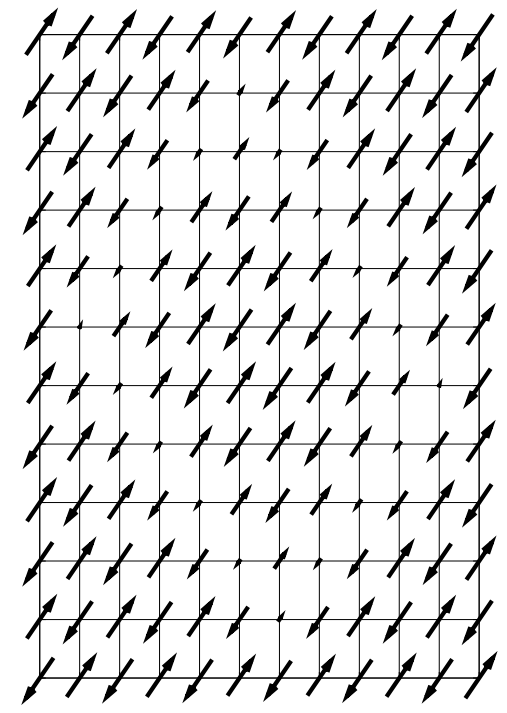

(a)

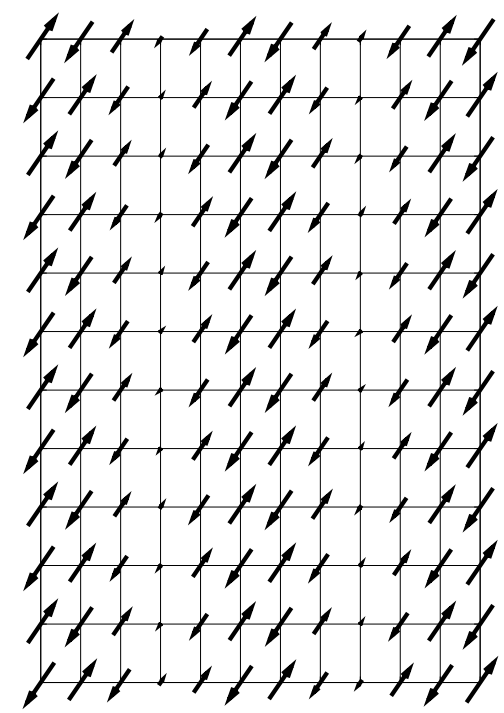

(b)

FIG. 7. Ground-state spin configuration for Hubbard models on a $12 \times 12$ cluster with $U=5$ and open BCs. (a) $x=1 / 8$, $t_{x}=t_{y}=-1$; corresponding charge distribution in Fig. 4(a). (b) $x=1 / 6, t_{x}=-0.9, t_{y}=-1.1$; corresponding charge distribution in Fig. 3(e). Length of largest spins represents $85 \%$ of full moment.

towards the non-uniform stripe phase.

A further qualitative point concerns the magnetic nature of the domain walls. As illustrated in Fig. 7, all of the corral and uniform stripe phases are found to have antiphase domain walls. Those sites located in the walls possess no magnetic moment, and the AF regimes on each side have a mutual phase shift of $\pi 19$.20 Fig. 7(a) shows the spin configuration for the diagonal corral state in Fig. 4(a), illustrating from the phase shift between the inside and outside of the corral that the diagonal domain wall, whose sites have no moment, can be considered to

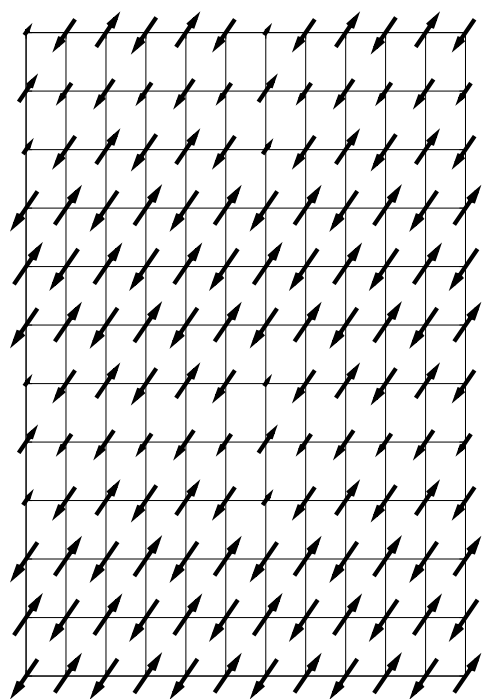

FIG. 8. Ground-state spin configuration for Hubbard model on a $12 \times 12$ cluster with $U=5, x=1 / 8$, periodic BCs and $t_{x}=-0.9, t_{y}=-1.1$; corresponding charge distribution in Fig. 4(a). Spin scale as in Fig. 7.

be filled, or uniform. Fig. 7(b) shows that the uniform stripe state in Fig. 3(e) possesses the same property. By contrast, we see in Fig. 8 for the non-uniform stripe state close to half-filling that there is no phase change ( $c f$. Fig. 1(c), Ref 29), meaning that the stripe is an in-phase domain wall. 36 This result is also opposite to that of the uniformy half-filled stripe, which is an antiphase domain wall 291 for the same reason as for the filled stripe. In the non-uniform case, the sites in the center of the stripe retain a magnetic moment, and these are ferromagnetically (FM) aligned. However, we believe this to be driven not by the preference for a FM hopping channel, but by the spin orientations of the sites surrounding those with higher hole densities: these sites are analogous to a missing electron, with charge and spin which reflect the hopping from the four nearest neighbors. The one-particle physics suggested by these observatigns supports an interpretation by Nagaoka's theorem.52 53 It appears that the non-uniform stripe could be considered as aligned, small FM polarons.

Within the limits of the HF approximation, we may quantify some of the above observations by inspecting the energies of the solutions. Table I illustrates the effect of increasing anisotropy on the total energy for one parameter set (Figs. 3(a), (c) and (e)) by decomposition into potential and kinetic energy components. We see that increasing anisotropy causes a small increase in the potential contribution (double-occupancy energy), but that this is more than compensated by gains in the kinetic part. The strong effect of the hopping anisotropy is clearly demonstrated by the fact that for a $22 \%$ anisotropy in $t$ values (right column), the kinetic energies differ by almost a factor of 2 . This strong increase in energy difference reflects the dominance of transverse 
TABLE I. Total ground-state energy $E_{\text {tot }}$, and kinetic $\left(E_{K}^{x}, E_{K}^{y}\right)$ and potential $\left(E_{P}\right)$ energy components per site for Hubbard model on a $12 \times 12$ cluster with $U=5, x=1 / 6$, open BCs and increasing anisotropy $t_{x} / t_{y}$; corresponding charge distributions in Fig. 3(a), (c) and (e).

\begin{tabular}{c|c|c|c}
\hline \hline & $t_{x} / t_{y}=1$ & $1.05 / 0.95$ & $1.1 / 0.9$ \\
\hline$E_{\text {tot }}$ & -0.78782 & -0.79361 & -0.80317 \\
$E_{K}$ & -1.22931 & -1.24634 & -1.25942 \\
$E_{K}^{x}$ & -0.61465 & -0.72917 & -0.80513 \\
$E_{K}^{y}$ & -0.61465 & -0.51717 & -0.45429 \\
$E_{P}$ & 0.44148 & 0.45273 & 0.45625 \\
\hline \hline
\end{tabular}

hopping in stabilizing the uniform stripe phase.

Table II shows the total energies per site for $12 \times 12$ clusters at hole dopings $x$ of $0,1 / 8$ and $1 / 6$, and Table III the differences between doped and undoped systems presented as energy per hole. From Table II, systems with periodic BCs have significantly lower total energy, but a rather similar energy per hole. The same evolution of the total energy with anisotropy as in Table I occurs for all parameter sets with open BCs, and also at half-filling $(x=0)$, for the same reason as above. However, with periodic BCs a small anisotropy appears in most cases to result in a higher overall energy, but larger anisotropy values restore the behavior observed with open BCs. This result we ascribe to the competition of different possible structures within the system before a truly 1d state may be established; periodic BCs vastly increase the degeneracy of available solutions, sometimes leading to convergence difficulties, and one has less confidence of finding the true ground state. Turning to Table III, the value of the energy per hole reflects both the mobility of the holes and the gain in potential energy due to a lower probability of double occupancy. These trends compete as $U$ is increased, and also as $x$ is increased, as a consequence of which the values in the table vary rather little; this statement applies also for smaller $(x=1 / 12)$ and larger $(x=1 / 4)$ doping levels (not shown).

In concluding this subsection, we have found that hopping anisotropy in a Hubbard model can give rise to two qualitatively different types of stripe state, whose appearance depends on the intrinsic system parameters, and whose nature is influenced by the commensurability of the filling with the cluster size. Uniform stripes have the same charge density and no magnetic moment on every site, align perpendicular to the direction of strong hopping, represent antiphase domain walls between AF regions on either side, and are energetically rather stable. Non-uniform stripes have alternating charge density and finite magnetic moment on the central sites, align in the direction of strong hopping, and act as in-phase domain walls. Both types of stripe are stabilized by hopping between sites of differing charge density, which in the uniform case means transverse hole hopping, but in the non-uniform case results in similar contributions from
TABLE II. Ground-state energies per site for Hubbard model on a $12 \times 12$ cluster. $\mathrm{O}$ and $\mathrm{P}$ denote open and periodic $\mathrm{BCs}$, anisotropies quoted as ratio $t_{x} / t_{y}$.

\begin{tabular}{c|c|c|c|c|c}
\hline \hline $\mathrm{BCs}$ & $U$ & $t_{x} / t_{y}$ & $x=0$ & $1 / 8$ & $1 / 6$ \\
\hline $\mathrm{O}$ & 5 & 1.00 & -0.6321 & -0.7514 & -0.7878 \\
$\mathrm{O}$ & 5 & 1.11 & -0.6340 & -0.7534 & -0.7936 \\
$\mathrm{O}$ & 5 & 1.22 & -0.6397 & -0.7563 & -0.8032 \\
$\mathrm{P}$ & 5 & 1.00 & -0.6820 & -0.7967 & -0.8325 \\
$\mathrm{P}$ & 5 & 1.11 & -0.6840 & -0.7905 & -0.8330 \\
$\mathrm{P}$ & 5 & 1.22 & -0.6902 & -0.7950 & -0.8551 \\
$\mathrm{O}$ & 8 & 1.00 & -0.4294 & -0.5550 & -0.5999 \\
$\mathrm{O}$ & 8 & 1.11 & -0.4306 & -0.5591 & -0.6008 \\
$\mathrm{O}$ & 8 & 1.22 & -0.4341 & -0.5624 & -0.6113 \\
$\mathrm{P}$ & 8 & 1.00 & -0.4659 & -0.5974 & -0.6429 \\
$\mathrm{P}$ & 8 & 1.11 & -0.4672 & -0.5971 & -0.6401 \\
$\mathrm{P}$ & 8 & 1.22 & -0.4710 & -0.6002 & -0.6453 \\
\hline \hline
\end{tabular}

TABLE III. Ground-state energies per doped hole for Hubbard model on a $12 \times 12$ cluster at fillings $x=1 / 8$ and $x=1 / 6$. $\mathrm{O}$ and $\mathrm{P}$ denote open and periodic BCs, anisotropies quoted as ratio $t_{x} / t_{y}$.

\begin{tabular}{c|c|c|c|c}
\hline \hline $\mathrm{BCs}$ & $U$ & $t_{x} / t_{y}$ & $x=1 / 8$ & $x=1 / 6$ \\
\hline $\mathrm{O}$ & 5 & 1.00 & -0.9539 & -0.9342 \\
$\mathrm{O}$ & 5 & 1.11 & -0.9553 & -0.9575 \\
$\mathrm{O}$ & 5 & 1.22 & -0.9328 & -0.9809 \\
$\mathrm{P}$ & 5 & 1.00 & -0.9176 & -0.9032 \\
$\mathrm{P}$ & 5 & 1.11 & -0.8502 & -0.8938 \\
$\mathrm{P}$ & 5 & 1.22 & -0.8385 & -0.9890 \\
$\mathrm{O}$ & 8 & 1.00 & -1.0052 & -1.0228 \\
$\mathrm{O}$ & 8 & 1.11 & -1.0284 & -0.9909 \\
$\mathrm{O}$ & 8 & 1.22 & -1.0268 & -1.0634 \\
$\mathrm{P}$ & 8 & 1.00 & -1.0523 & -1.0619 \\
$\mathrm{P}$ & 8 & 1.11 & -1.0391 & -1.0376 \\
$\mathrm{P}$ & 8 & 1.22 & -1.0336 & -1.0461 \\
\hline \hline
\end{tabular}

both longitudinal and transverse hole motion.

\section{E. Variation of extrinsic parameters}

In the preceeding subsections we have already noted some of the effects of the extrinsic parameters, a term we use to refer to cluster dimensions, BCs, and commensuration between filling and cluster size. Open BCs cause a definite tendency for holes to avoid the edges, where their hopping energy is lowered. While for small doping this merely sets the inhomogeneous charge structure in the center of the cluster, at larger dopings it can act to influence the alignment of domain walls (Fig. 3(a)), particularly at smaller values of $U$ where the charge structures are extended. Periodic BCs relax this constraint, and thus facilitate the formation of domain lines with the optimal orientation (diagonal (Fig. 5(a)) or vertical (Fig. 4(d), (f), also as in Fig. 3(e))) for the 


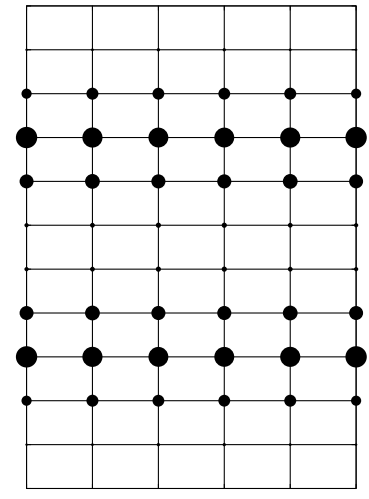

(a)

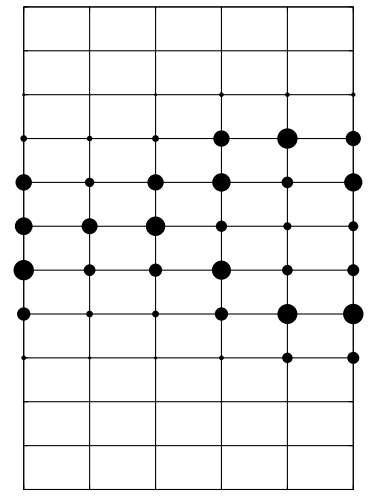

(b)
FIG. 9. Ground-state charge distribution for Hubbard models on a $12 \times 6$ cluster with $U=5$ and $t_{x}=t_{y}=-1$. (a) $x=1 / 6$, open BCs. (b) $x=1 / 8$, periodic BCs. Charge scale as in Fig. 3.

anisotropy, and clusters of the optimal size and configuration (Figs. 4(b), 5(a)). These effects are also inseparable from the issue of the commensuration between the cluster size and the number of doped holes, which is instrumental in determining the type of the polaron lattice (Figs. 4(b), 5(a)), and the filling and nature of the stripes (Figs. 3(e), 4(f)), in the cases where these form. As in all such studies on finite clusters, the possibility arises of large, finite-size gaps in the quasiparticle spectrum (for example, between fillings of 24 and 25 holes in the $12 \times 12$ system). However, we do not expect that such effects would particularly favor one class of chargeinhomogeneous solution over another.

A more primitive effect of cluster size emerges when at least one of the dimensions becomes small on the scale of the equilibrium, inhomogeneous charge structure. This is illustrated in Fig. 9(a) for a $12 \times 6$ system with hole doping $x=1 / 6$. Although the intrinsic parameters are the same as in Fig. 3(a), the inhomogeneous charge structure of minimum energy in this system is a pair of antiphase domain walls, even for open BCs. This observation should be borne in mind when interpreting the results of small-system studies such as exact diagonalization and DMRG, in which rather more sophisticated analyses are necessary to establish the true ground state of a hypothetical infinite system. Fig. 9(b) shows the analogous result for $x=1 / 8$, where the 9 holes form one and a half stripes with periodic BCs.

Finally, the nature of the ground state is very strongly influenced by the choice of periodic or antiperiodic BCs. This is more readily implemented by retaining periodic BCs, but working on a cluster with one side chosen to be odd, and was the method by which Zaanen and Gunnarsson 19 first observed stripe solutions in the form of a single domain wall. The analogous result for systems even on both sides was a closed domain wall like the corrals presented above, but the straight domain line was found to offer the minimum energy per particle. We have also considered even $\times$ odd and odd $\times$ odd systems with both periodic and mixed BCs (open in the odd direction, periodic in the even 31 ), and in the same way find the possibility of "trapping" stripe solutions in the form of single domain walls. Such studies are useful in the context of investigating the effects of anisotropy, as one may monitor the total energy of the fixed configuration as a function of $t_{x} / t_{y}$, and we will employ this approach below for the $t-J$ model. However, for a variety of reasons we do not consider single, infinite domain walls to be viable solutions for the isotropic system in the thermodynamic limit, and so have chosen not to dwell upon this type of analysis.

For any 3d system of weakly coupled planes, longrange $\mathrm{AF}$ order is favored if the domain walls form closed loops 31 We note in our studies a preference for domain lines in corrals to lie diagonally, in accordance with expectation for nearest-neighbor hopping, but that in no cases did a diagonal stripe phase appear with periodic BCs. Instead we find a preference for small, diagonal polarons rather than extended lines in the isotropic system, and that vertical stripes required the LTT-inspired hopping anisotropy. A further qualitative observation in this direction is the absence in our isotropic, even $\times$ even studies of a phase with two parallel, vertical domain walls, even at the optimal filling for this state (cf. Fig. 3(e)). With the goal of elucidating the effects of anisotropy on the static solutions, we have concentrated on the most unbiased initial conditions which appear to retain the possibility of sampling the candidate ground states.

\section{V. $t-J$ MODEL}

We turn now to anisotropy effects in the $t$ - $J$ model (8). The previous section has given considerable insight into the complex range of possibilities which may arise. $\mathrm{Nu}-$ merical studies of the $t-J$ model 31 have provided evidence for charge structures of closed domain-wall loops similar to those we find in the Hubbard model, but also for a close competition with many other structures, including open lines (stripes). In order to assess anisotropy effects, and to address the question of collaboration or competition between $t$ and $J$ in stripe formation and alignment, it will be necessary to characterize the favorability in different regimes of $2 \mathrm{~d}$ vs. $1 \mathrm{~d}$ structures, corrals vs. polarons, in-phase vs. antiphase dgmain lines, and the possible role of phase separation. 5456

In the real-space HF approximation to the $t-J$ model one encounters an immediate technical problem. The value of $U$ shold be very large (Sec. III), in order to have the Hubbard term act to project out any states with doubly-occupied sites from the space of those considered. In a selection of studies with $U=100$, we found the results to vary appreciably with changes in $J$, and 


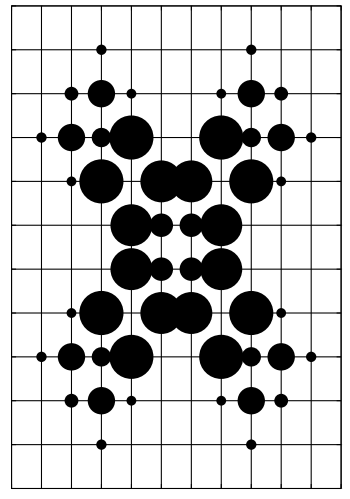

(a)

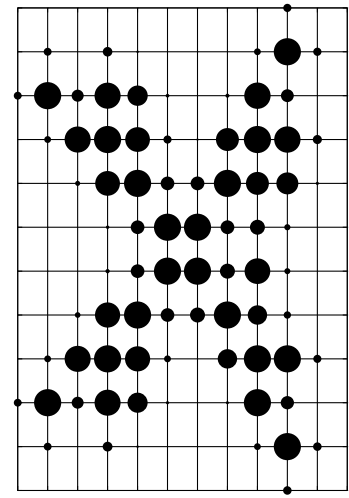

(b)

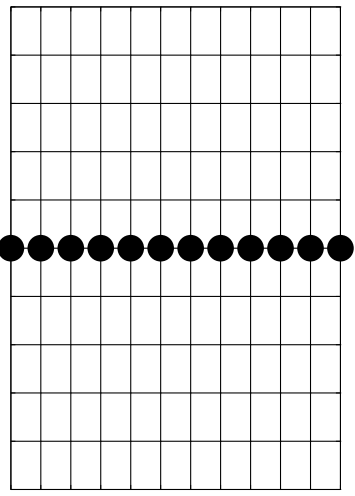

(a)

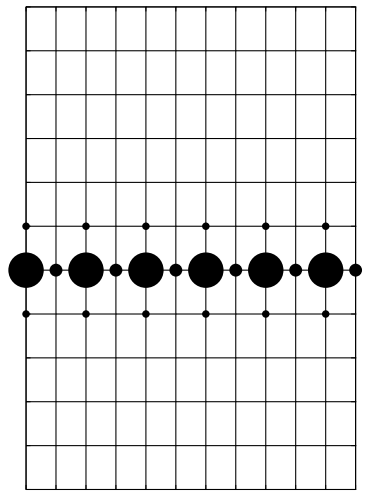

(b)
FIG. 10. Ground-state charge distribution for Hubbard models on a $12 \times 12$ cluster with $x=1 / 6$, open BCs, and $t_{x}=t_{y}=-1$. (a) $U=100$. (b) $U=1000$. Charge scale as in Fig. 3. Spin distributions (not shown) are AF with unaltered phase.

particularly with changes of $J_{x}$ relative to $J_{y}$, on the order of 0.05 . The "residual" superexchange term in a pure Hubbard model, $J=4 t^{2} / U=0.04$ for this choice of $U$, suggests that still larger values of $U$ are required for a degree of projection which can safely be taken to represent the asymptotic limit. This observation is supported by computing the value of the double-occupation energy. Indeed, as shown in Fig. 10, while a Hubbard model with $U=100$ gives a robust, polaronic state with variations of charge between sites, the situation for $U=1000$ (and larger) corresponds to a cluster of uniformly charged sites more reminiscent of incipient phase separation, and with no obvious contribution from kinetic terms. We note also in connection with Fig. 10(b) that the calculation has converged to a state lacking the fourfold symmetry expected from a uniform initial configuration, which is indicative of difficulties in employing the real-space $\mathrm{HF}$ technique in this parameter regime.

For such large values of $U$, (i) every local minimum becomes very deep $(\mathcal{O}(U))$, and (ii) the fluctuation energies $\left(\mathcal{O}\left(t^{2} / U\right)\right)$ become very small. Calculations in this regime show that the method loses its flexibility to find a global minimum, and that the final state is determined largely by the chosen starting configuration. Physically, large $U$ projects out the relevance of quantum fluctuations, a statement we will make more specific in the following example. There remain two possible courses of action by which one may gain insight into the anisotropic $t-J$ model using the real-space HF approach. As outlined in the preceeding section, the first is to create a captive stripe at large $U$, and to study through the ground-state energy $E_{\text {tot }}$ the qualitative question of how anisotropies in $t$ and $J$ affect such a state. The second is to reduce the value of $U$ to a size at which the system appears once again to have the ability to explore a number of distinct
FIG. 11. Ground-state charge distribution for $t$ - $J$ models with periodic BCs, $t_{x}=t_{y}=-1, J_{x}=J_{y}=0.35$, and $U=1000$. (a) $12 \times 11$ cluster with 12 holes, circle radius on stripe represents $98.1 \%$ hole occupation; (b) $12 \times 12$ cluster with 6 holes, circle radii on stripe represent $55.9 \%$ and $20.3 \%$ hole occupation.

states, and to seek indications for the validity of the results in a model with true projection.

\section{A. Large $U$}

In this subsection we consider the kinetic and magnetic energies of filled and half-filled stripes as a function of the anisotropy. We set $U=1000$ to ensure adequate removal of doubly occupied sites, and consider the canonical $t$ $J$ model parameter ratio $J / t=0.35$. We trap stripes of 12 and 6 holes along the center line of a $12 \times 11$ and $12 \times 12$ clusters, respectively, with periodic BCs, and compute the kinetic and magnetic energies of the system with $\pm 11 \%$ anisotropy in both $t$ and $J$. Because the stripe is aligned along the $\hat{x}$-direction, automatically breaking any 4-fold symmetry, positive and negative anisotropy effects are not necessarily the same. We find that the schematic picture of the fully local stripe is very nearly realised (to $98 \%$, Fig. 11(a)) in the uniform case (12 holes) when the spin configuration is set such that the stripe is an antiphase domain wall, as in the previous section. Similarly, the half-filled stripe solution (Fig. 11(b)) emerges only when the stripe is an in-phase domain wall, which accounts for the different transverse cluster dimensions used in each case. Thus we do not show the corresponding spin distributions for Fig. 11, which consist simply of full-moment AF regions on both sides of the stripe, with (a) and without (b) a phase shift across the domain wall.

The results for kinetic and magnetic energies are presented in Tables IV and V. We list these separately because the numbers are not directly comparable at large $U$ : while the magnetic energy is determined essentially 
TABLE IV. Kinetic (upper figure) and magnetic (lower) energy components per site for $t-J$ model on a $12 \times 11$ cluster with 12 holes, periodic BCs, and $U=1000$.

\begin{tabular}{c|c|c|c}
\hline \hline & $t_{x}=-0.95$ & $t_{x}=-1$ & $t_{x}=-1.05$ \\
& $t_{y}=-1.05$ & $t_{y}=-1$ & $t_{y}=-0.95$ \\
\hline$J_{x}=0.3325$ & -0.045534 & -0.043226 & -0.040996 \\
$J_{y}=0.3675$ & -0.30086 & -0.30088 & -0.30090 \\
\hline$J_{x}=0.35$ & -0.045512 & -0.043206 & -0.040976 \\
$J_{y}=0.35$ & -0.30163 & -0.30165 & -0.30167 \\
\hline$J_{x}=0.3675$ & -0.045491 & -0.043185 & -0.040955 \\
$J_{y}=0.3325$ & -0.30239 & -0.30241 & -0.30243 \\
\hline \hline
\end{tabular}

TABLE V. Kinetic (upper figure) and magnetic (lower) energy components per site for $t-J$ model on a $12 \times 11$ cluster with 6 holes, periodic BCs, and $U=1000$.

\begin{tabular}{c|c|c|c}
\hline \hline & $t_{x}=-0.95$ & $t_{x}=-1$ & $t_{x}=-1.05$ \\
& $t_{y}=-1.05$ & $t_{y}=-1$ & $t_{y}=-0.95$ \\
\hline$J_{x}=0.3325$ & -0.089029 & -0.088635 & -0.088445 \\
$J_{y}=0.3675$ & -0.31249 & -0.31261 & -0.31272 \\
\hline$J_{x}=0.35$ & -0.089018 & -0.088626 & -0.088438 \\
$J_{y}=0.35$ & -0.31255 & -0.31267 & -0.31280 \\
\hline$J_{x}=0.3675$ & -0.089006 & -0.088615 & -0.088429 \\
$J_{y}=0.3325$ & -0.31260 & -0.31275 & -0.31289 \\
\hline \hline
\end{tabular}

by $J$, and depends only on the number of antiparallel spin pairs, the kinetic energy requires a transfer of electrons between sites. Although a transverse hopping would appear to be favorable between neighboring filled and empty sites, the expectation value $\left\langle c_{i \sigma}^{\dagger} c_{j \sigma}\right\rangle$ depends on the component of the final-state configuration present in the HF wave function (which should be considered as a linear superposition of many charge configurations in the basis of lattice sites). Large values of $U$ act to suppress these additional components, and thus the expectation values $\left\langle E_{K}\right\rangle$. This is the sense in which quantum fluctuations are projected out, and it is this feature which we will restore in the following subsection.

Comparing the kinetic energies for the uniform stripe configuration (Table IV, Fig. 11(a)), we find little variation with $J$, and a strong preference for larger transverse hopping $t_{y}$. Thus the effect of hopping anisotropy is as in the Hubbard model. Quantitatively, that $11 \%$ anisotropy leads to a $5 \%$ energy change, in contrast to the $30 \%$ effect in the Hubbard model with periodic BCs, can be ascribed to the fact that the $1 \mathrm{~d}$ state is already established in the isotropic case. The magnetic energies show a negligible dependence on the hopping, and a rather small overall effect which is due to the large AF regions undisturbed by the presence of the stripe. However, the variation clearly indicates a preference for alignment of the stripe with the direction of stronger superexchange, a result readily explained at zeroth order: the stripe cuts two bonds in the $\hat{y}$-direction, and only one in $\hat{x}$, so clearly costs less mag- netic energy in longitudinal alignment. Thus for filled stripes in the $t$ - $J$ model the two anisotropies compete. A detailed analysis by techniques better able to account for quantum fluctuation effects 31.32 is clearly required to quantify this competition.

Results for the half-filled, in-phase stripe (Fig. 11(b)) are given in Table V. The variation in kinetic energies with anisotropy is remarkably small in comparison with the uniform stripe, and it would appear fair to say that leading-order hopping contributions along the chain are cancelled by transverse hopping (primarily to the sites of larger hole density) for the ideal configuration established here. These results present no evidence to support alignment of the alternating stripe with the direction of strong hopping, and in fact show a weak preference for transverse alignment. The variation in magnetic energies with anisotropy is similarly weak, as expected from the bond-counting argument. Corrections arise from the fact that the stripe now possesses a FM center line ( $c f$. Fig. 9), and tend also to favor transverse alignment. The orientation of half-filled stripes is thus a delicate issue, and we are unable to make any firm statements on the basis of these results. For the same reason we have not tried to address further interesting questions which may be posed in this context, such as the energetics of corners in domain walls, crossings of stripes, or interactions between stripes placed in proximity to each other. 19 Finally, for both types of stripe we note that there is negligible interplay between the individual effects of anisotropies in $t$ and $J$, with energy changes proceeding linearly and separately in both variables.

\section{B. Intermediate $U$}

In this subsection we will show results obtained in the spirit of setting $U$ as large as possible while retaining the possibility of finding the true ground state. This is the approach adopted in Ref. 25, and enables one to restore the competition between kinetic and magnetic energies in examining the above effects, but introduces a different set of questions concerning the physics of the $t-U-J$ model. .7

We begin by characterizing the effects of $J$ in the isotropic model with $U=50$. Fig. 12 shows the evolution of the ground-state charge distribution as $J$ is increased. Even rather small values of the superexchange interaction alter the $J=0$ structure (Fig. 12(a)) to one with stronger charge differentiation between and within small clusters (Fig. 12(b), also realized for $J=0.05$ ). At intermediate values of $J$ there is evidence of competition with a phase (not shown) of small polarons showing preferential diagonal alignment. As $J$ is increased towards the canonical cuprate-model value of 0.3 , the small- $J$ polaronic phase of Fig. 12(b) exhibits increasing spacing of the charge clusters, or preference for undisturbed $\mathrm{AF}$ regions (Fig. 12(c)), and shortly beyond this we find separated clumps of uniformly distributed charges 


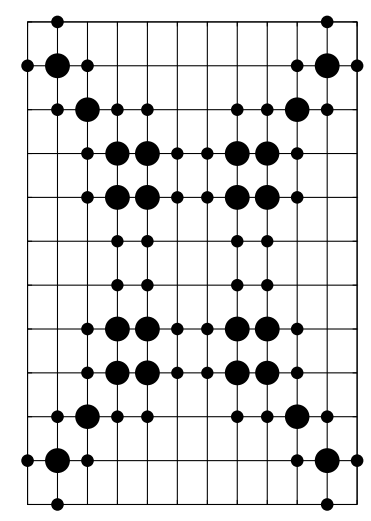

(a)

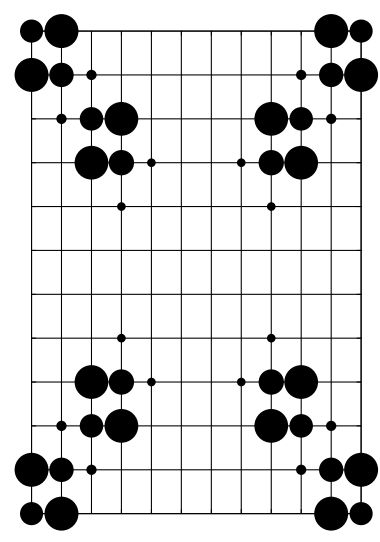

(c)

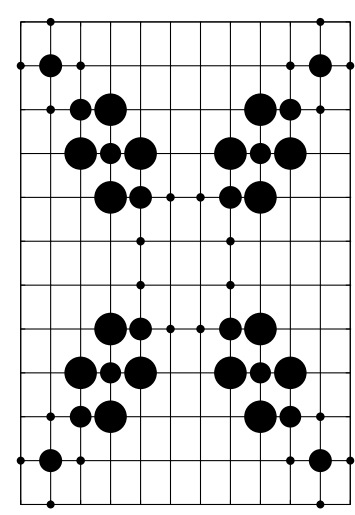

(b)

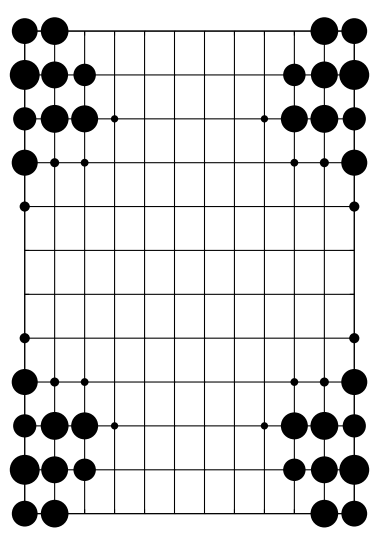

(d)

FIG. 12. Ground-state charge distribution for $t-U-J$ model on a $12 \times 12$ cluster with open BCs, for hole doping $x=1 / 6$, $U=50$, and $t_{x}=t_{y}=-1$. (a) $J_{x}=J_{y}=0$. (b) $J_{x}=J_{y}=0.1$. (c) $J_{x}=J_{y}=0.3$. (d) $J_{x}=J_{y}=0.5$. Charge scale is such that largest circles represent $75.1 \%$ hole occupation. Spin distributions (not shown) are AF with unaltered phase.

(Fig. 12(d)) in a phase which we regard as representative of true phase separation. The quantitative value at which this separatipn occurs is not unexpected from previous estimates 5456

With periodic BCs this tendency to phase separation is strengthened, but so also is the tendency to formation of anisotropic structures in the presence of hopping anisotropy (below). In certain parameter regimes it appears possible for this anisotropy to suppress phase separation, which in a systematic study would lead to moving the phase boundary to higher $J$. By contrast, anisotropy in the superexchange has little effect at small $J(\lesssim 0.1)$, where the system remains largely dominated by kinetic processes, but appears to promote the phase separation.

In this parameter regime the spin state is not altered from its starting configuration of very weak antiferromagnetism, which is simply reinforced during the selfconsistency procedure. This maintenance of the spin con-

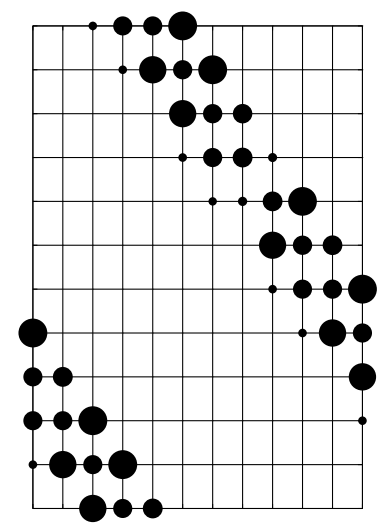

(a)

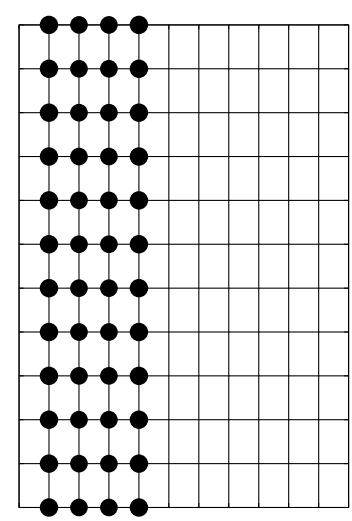

(b)
FIG. 13. Ground-state charge distribution for $t-U-J$ model on a $12 \times 12$ cluster with periodic BCs, for hole doping $x=1 / 6, U=100$, and $J_{x}=J_{y}=0.2$. (a) $t_{x}=t_{y}=-1$. (b) $t_{x}=-1.1, t_{y}=-0.9$. Charge scale is such that largest circles represent $75.5 \%$ hole occupation.

figuration is not an artefact of considering only a single spin component (Sec. III), as retaining all three components returns the identical result expected from spin rotation symmetry. While the resulting charge inhomogeneities have in-phase nature, we cannot exclude the possibility that more extended structures ( $c f$. Sec. IV), which reverse the spins over a finite region, form a separate class of minima inaccessible from this initial state.

Fig. 13(a) illustrates this point with a result obtained in an isotropic system with periodic BCs and $U=100$. The emergence of a diagonal stripe is a consequence of the commensurate filling, and in fact the structure should more correctly be described as a "bistripe". The possibility offered by this filling to create a wall with a $2 \pi$ phase rotation (Fig. 14) allows this configuration to exist within an otherwise in-phase, AF background. Hopping anisotropy for this parameter choice leads to a rather different form of bistripe (Fig. 13(b)), whose orientation is changed to vertical even by rather small differences between $t_{x}$ and $t_{y}$. As noted above, superexchange anisotropy $J_{x} \neq J_{y}$ promotes polaron formation and phase separation of the above kind (Fig. 12(f)); quantitatively, $11 \%$ anisotropy at $J=0.3$ (cf. Fig. 12(c)) and $22 \%$ at $J=0.1$ (cf. Fig. 12(b)) are sufficient to drive the separation. Finally, the qualitative results described in this section depend rather little on the chosen value of the "large" $U$, which was varied between 16 and 100 .

We return now to the question of the competition between the stripe alignment tendencies of anisotropies in $t$ and $J$. The most unbiased approach we can find to investigate this is to set $U=16$, and to begin with a uniform charge distribution, and zero initial spin on all sites except those in two corners. The following results are presented for a $12 \times 11$ cluster with periodic BCs, 


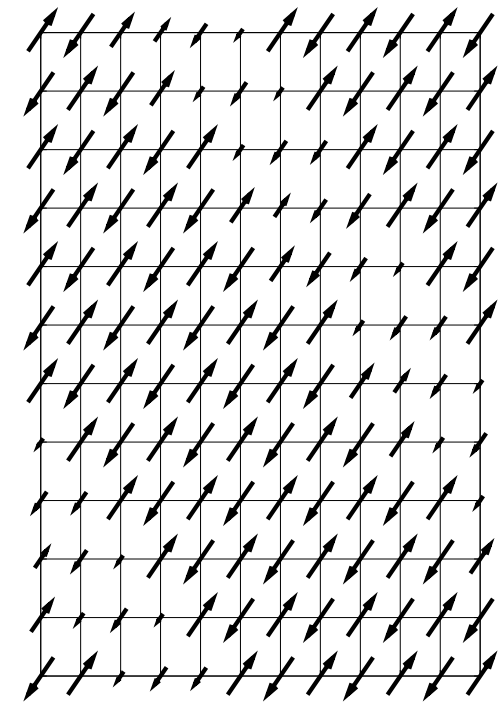

FIG. 14. Ground-state spin configuration for $t-U-J$ model on a $12 \times 12$ cluster with periodic BCs, for hole doping $x=1 / 6, U=100, t_{x}=t_{y}=-1$, and $J_{x}=J_{y}=0.2$; corresponding charge distribution in Fig. 13(a). Spin scale as in Fig. 7.

which we have chosen to favor stripes of the experimental orientation. We used $J=0.5$, as in Ref. 25, but found little qualitative difference at $J=0.25$ despite the results of Fig. 12. In Fig. 15(a) is shown the charge distribution for parallel spins at diagonally opposite corners of the cluster, which for the chosen dimensions sets the requirement of an antiphase domain wall. Indeed a domain wall is formed, but despite the commensurate filling (12 holes) it is not straight, and wandering of the domain line becomes more pronounced at smaller $J$, such that the line sections are closer to being diagonal. The competition between a preference for diagonal domain walls from nearest-neighbor hopping and vertical domain walls from superexchange is exactly that expected from the simplest considerations on the idealized stripe.

In Fig. 15(b) is shown the result of setting an in-phase configuration across the cluster: the holes prefer to form a diagonal domain line, and a wandering one whose local direction is again mostly diagonal. On periodic continuation of the cluster, the line appears as a sawtooth. The prevalence of kinetic terms over magnetic in the charge alignment is not apparent on the basis of Fig. 12, but we note that the doping here is much lower. In this case there is a reversal of the AF spin configuration across the domain wall, a result which argues for initial configurations containing a single "seed" spin, from which all other orientations may be "grown". In fact such an approach produces few qualitative differences from the results presented in this section, where the starting configuration was weakly AF. For smaller values of $U$ the system is sufficiently flexible to choose the appropriate configuration, and for larger $U$ the result of the "growth" procedure is either a rather more diffuse version of the same

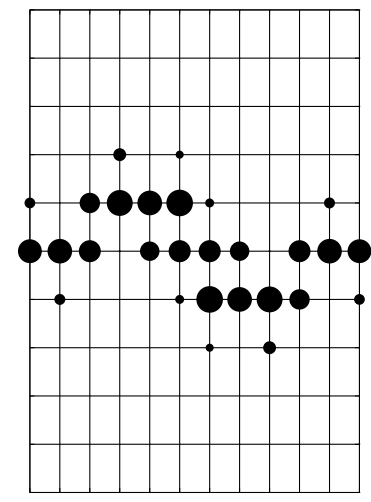

(a)

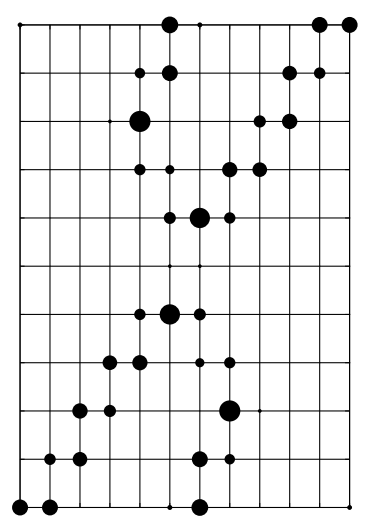

(b)
FIG. 15. Ground-state charge distribution for $t-U-J$ model on a $12 \times 11$ cluster with periodic BCs, for 12 holes $(x=1 / 11)$, $U=16, t_{x}=t_{y}=-1$, and $J_{x}=J_{y}=0.5$. Initial configuration with upper left and lower right spins (a) parallel and (b) opposite. Charge scale as in Fig. 3. Spin distributions (not shown) are AF with $\pi$ phase shifts across all domain walls.

charge structure (with higher net energy), or a random arrangement indicating the local-minimum problem.

Variations of the $t$ - and $J$-anisotropies on the two states in Fig. 15 have little visible effect on the charge configurations even at the $22 \%$ level, although in Fig. 15(b) there is noticeable charge redistribution to a more "vertical" local stripe orientation. Thus we revert to analyzing the energies of the ground states, which are presented in Tables VI and VIII in the same format as in Tables IV and V. It is immediately clear that kinetic and magnetic energies are of the same order again in the regime around $U=16$, and it becomes meaningful to consider their combination $E_{\text {tot }}$. The next striking feature of the data in Table VI is the remarkably small change in the magnetic energy $E_{m}$ as a function of $J_{x} / J_{y}$ : despite the fact that the system appears to contain a stripe, and has no frustrated bonds at the boundaries, variation of $E_{m}$ with $J_{x} / J_{y}$ is in fact weaker than with $t_{x} / t_{y}$. Another interesting property is that the kinetic energy decreases for both types of hopping anisotropy, a result which would not be expected for a vertical stripe.

The explanation for both of these features is contained in Table VII, which shows the components of $E_{K}$ and $E_{m}$ for the $\hat{x}$ - and $\hat{y}$-directions separately. The magnetic energies (lower figures) vary linearly with $J_{x}$ and $J_{y}$, with their sum (Table VI) almost a constant quantity. The kinetic energies change very strongly with $t_{x}$ and $t_{y}$, as already observed in Table I, but again their sum changes only weakly. It is clear from the figures that the stripe in Fig. 15(a) is almost perfectly diagonal in character. The bond-counting argument for effects of $J$ on stripe orientation give no preference in the case of a diagonal stripe, and show how well this picture works even for a system 
TABLE VI. Kinetic (upper figure) and magnetic (lower) energy components per site for $t$ - $J$ model on a $12 \times 11$ cluster with 12 holes, periodic BCs, $U=16$, and a starting configuration of parallel spins on opposite corners; charge distribution in Fig. 15(a).

\begin{tabular}{c|c|c|r}
\hline \hline & $t_{x}=-0.9$ & $t_{x}=-1$ & $t_{x}=-1.1$ \\
& $t_{y}=-1.1$ & $t_{y}=-1$ & $t_{y}=-0.9$ \\
\hline$J_{x}=0.45$ & -0.56846 & -0.55757 & -0.56347 \\
$J_{y}=0.55$ & -0.40620 & -0.40098 & -0.39812 \\
\hline$J_{x}=0.5$ & -0.56789 & -0.55738 & -0.56349 \\
$J_{y}=0.5$ & -0.40712 & -0.40160 & -0.39848 \\
\hline$J_{x}=0.55$ & -0.56733 & -0.55718 & -0.56350 \\
$J_{y}=0.45$ & -0.40809 & -0.40228 & -0.39888 \\
\hline \hline
\end{tabular}

TABLE VII. Energy components $\left\langle E_{K}^{x}\right\rangle,\left\langle E_{K}^{y}\right\rangle,\left\langle E_{m}^{x}\right\rangle$, and $\left\langle E_{m}^{y}\right\rangle$ per site for $t-J$ model on a $12 \times 11$ cluster with 12 holes, periodic BCs, and $U=16$.

\begin{tabular}{l|c|c|r}
\hline \hline & $t_{x}=-0.9$ & $t_{x}=-1$ & $t_{x}=-1.1$ \\
& $t_{y}=-1.1$ & $t_{y}=-1$ & $t_{y}=-0.9$ \\
\hline & -0.20022 & -0.26859 & -0.33180 \\
$J_{x}=0.45$ & -0.36824 & -0.28898 & -0.23167 \\
$J_{y}=0.55$ & -0.18584 & -0.18201 & -0.17987 \\
& -0.22036 & -0.21896 & -0.21825 \\
\hline & -0.20045 & -0.26917 & -0.33278 \\
$J_{x}=0.5$ & -0.36745 & -0.28820 & -0.23070 \\
$J_{y}=0.5$ & -0.20678 & -0.20255 & -0.20010 \\
& -0.20034 & -0.19905 & -0.19838 \\
\hline & -0.20066 & -0.26975 & -0.33376 \\
$J_{x}=0.55$ & -0.36667 & -0.28743 & -0.22973 \\
$J_{y}=0.45$ & -0.22778 & -0.22313 & -0.22037 \\
& -0.18031 & -0.17914 & -0.17852 \\
\hline \hline
\end{tabular}

with a broad charge distribution far from the idealized stripe. Changes in magnetic energy due to contributions from the hopping term are on the $1 \%$ level. The energy gain for both anisotropy directions also requires a predominantly diagonal nature, although the minor preference for strong $t_{y}$ indicates that the stripe does preserve a small amount of $(1,0)$ character (less than $5 \%$ compared to the changes in $x$ - and $y$-components). DMRG studies of the $t-J$ model also show a significant presence of diagonal stripes, and their near-degeneracy in energy with vertically oriented domain walls. 11

This result is rather surprising in view of the properties observed earlier in the Hubbard model, and invite speculation on the relationship of $t-U$ and $t-U-J$ models. A discussion of the energetics is incomplete without noting that on the same scale as in Tables VI-VIII the double-occupancy energy for $U=16$ is approximately 0.2 in every case, so is a quantity which may not be neglected. By contrast, in the previous subsection the double-occupancy energy for $U=1000$ was on the order of 0.0036 in each case.

Table VII shows the kinetic and magnetic energies un-
TABLE VIII. Kinetic (upper figure) and magnetic (lower) energy components per site for $t-J$ model on a $12 \times 11$ cluster with 12 holes, periodic BCs, $U=16$, and a starting configuration of antiparallel spins on opposite corners; charge distribution in Fig. 15(b).

\begin{tabular}{c|c|c|r}
\hline \hline & $t_{x}=-0.9$ & $t_{x}=-1$ & $t_{x}=-1.1$ \\
& $t_{y}=-1.1$ & $t_{y}=-1$ & $t_{y}=-0.9$ \\
\hline$J_{x}=0.45$ & -0.55432 & -0.56027 & -0.56816 \\
$J_{y}=0.55$ & -0.35716 & -0.38007 & -0.38864 \\
\hline$J_{x}=0.5$ & -0.55126 & -0.55928 & -0.56619 \\
$J_{y}=0.5$ & -0.35322 & -0.37394 & -0.38890 \\
\hline$J_{x}=0.55$ & -0.55414 & -0.55929 & -0.56597 \\
$J_{y}=0.45$ & -0.35327 & -0.37393 & -0.38927 \\
\hline \hline
\end{tabular}

der anisotropy changes of $22 \%$ in $t$ and $J$ for the charge configuration of Fig. 15(b). Once again, the values are comparable, and they are also very close indeed to the figures in Table VI for a very different charge configuration; in fact it is the magnetic energy which would drive a preference for the broad, meandering stripe of Fig. 15(a). In Fig. 15(b) there appears to be a higher net alignment of the domain walls along the $\hat{y}$-direction, and this may be taken to explain the preference shown by the kinetic energy for stronger $t_{x}$. Here we notice a rather strong effect of the hopping on the magnetic energy, for which the bond-counting argument also provides an explanation. In narrow stripes, a diagonal orientation breaks four bonds per site while a vertical one breaks three, and thus a hopping anisotropy (of either direction) which pushes a diagonal stripe towards a vertical one to gain transverse hopping energy will also lower the magnetic energy. That the net magnetic energy is lower for stronger $J_{y}$ is also consistent with the inferred $(0,1)$ character. The fact that the numbers in the lower two rows match to very high accuracy is not an error, as shown by the fact that the separate $x$ - and $y$-components of the relevant energies (not presented here) have the same $20 \%$ and $40 \%$ differences as in Table VII, and rather reflects the delicate nature of the energy balance for the weakly oriented domain-wall structure.

We have also considered the same calculations with different values of $U(=50)$ and of $J(=0.25)$, and find qualitatively similar results. Changing the filling $x$ also leaves the general conformation unaltered, although the domain wall loops in Fig. 15(b) become shorter. The analysis for an $11 \times 11$ system returns the same spin configurations as in Ref. 25, although we find the 12-hole domain line to be rather broad. For $12 \times 12$ arrays the domain lines tend to close, to avoid disturbing BCs which are already satisfied.

We close this section with a summary of the robust conclusions which may be drawn from our studies of the $t-J$ model. Without considering anisotropy, the superexchange term favors polarons over corrals. The presence of $J$ also leads to a form of phase separation, namely the formation of charge clumps near the edges of an open 
system. As a result, in the HF approximation, only systems with rather small values of $J$ may show formation of domain walls, whose alignment tends to be diagonal. This tendency is particularly strong in the $t-U-J$ model with intermediate values of $U$. Hopping anisotropy $t_{x} \neq t_{y}$ promotes uniform stripes normal to the strong hopping direction, as in the Hubbard model. There is a parameter regime of intermediate $J$ which favors small polarons, but no clear sign of the non-uniform, in-phase stripes observed for the Hubbard model. Superexchange anisotropy $J_{x} \neq J_{y}$ favors alignment of uniform stripes in the direction of stronger exchange, and thus competes with a hopping anisotropy of the same orientation. While quantitative results are hard to obtain, energetic considerations suggest that the relative effects of $t$ anisotropy are stronger. For the boundary region of $(t, J, x)$ parameter space between polaronic states and phase separation, we find that anisotropies in $t$ and $J$ act to stabilize coherent structures.

\section{DISCUSSION AND CONCLUSION}

In the previous sections we have detailed at length the many degrees of freedom allowed by the real-space $\mathrm{HF}$ approach, and the ways in which we use and interpret these to draw meaningful conclusions within this framework. We have also reviewed the experimental situation, focusing on those parts we are able to address, and have compared our results with existing theoretical literature. In this section we give a brief discussion of the more general context of our analysis.

In Sec. II we noted that the LTT distortion of the LSCO system provides anisotropies of 1-3\% in the hopping and superexchange parameters.47 Our investigations in Secs. IV and V required anisotropies on the order of $10-20 \%$, and these were not always sufficient to create a $1 d$ state. We appeal here to the quantitative insufficiencies of the HF technique to argue that our results indeed suggest an important role for anisotropies, as suggested by the comparison of the hopping energy difference with other key energy scales. The superconducting $T_{c}$, and the ordering temperature $T_{\mathrm{ch}}$ of stripe formation, are both around $40 \mathrm{~K}$ in LSCO. One of the very few indisputable results from the extensive studies of cuprate models is that there exist many competing candidate ground states whose energies are remarkably similar, and it seems not unreasonable that small anisotropies may play a significant role in selecting among these. In addition to the more detailed energetic considerations and analytical or numerical approaches which we mention below, a more accurate assessment of anisotropy effects would also require moving from a one-band model to the three-band case. 19 This step is also a prerequisite for discussing at the microscopic level the issue of bond- or site-centered stripes 31, 37 which is one we have not mentioned hitherto. All of the charge structures we find by unrestricted HF for the one-band model, with and without anisotropy, are site-centered, and we do not regard the model as appropriate for addressing this question.

The connection between the lattice structure and anisotropies in the electronic system suggests the importance of the phonon degrees of freedom. While dynamical phonons may play a significant role around the structural phase transition, the static distortion requires a slow phonon mode which becomes soft. Although static, averaged phonon variables have been included in some HF analyses 29 we remain unaware of any studies where the coupled phonon modes which are introduced have allowed the possibility of a spontaneous anisotropy. As noted in Sec. II, we have studied only a model with fixed anisotropy set by the lattice, and considered the energetics of the electronic system. When the structural transition is in close proximity to the electronic one, the need for a coupled model becomes apparent. The relevance of lattice energies for the electronic system may also be clarified by detailed experimental analysis of the energetics of the structural transition.

Other transition-metal oxide systems offer a clear example of the coupling of lattice and electronic degrees of freedom. The first observations of charge-ordering in stripe-like structures were made on nickelates, where the stripes are found to be diagonal (aligned along $(1,1)$ ). Detailed characterization of the ordering transition suggests once again a connection to the orthorhombic lattice structure arising from octahedral tilting at dopings $x<0.258$ Above this doping, charge ordering is a coupled structural and electronic event, which occurs at a single temperature, $T_{d}$, and this behavior persists to very high dopings $\left(\mathrm{La}_{2-x} \mathrm{Sr}_{x} \mathrm{NiO}_{4}\right.$ with $\left.x \leq 0.5\right)$. 9 In this case the energy change of the electronic system on adopting an anisotropic structure must be balanced directly against the distortion energy of the lattice, and theoretical estimates of the competing contributions become more involved. In manganite systems, the most common structural deformation is a Jahn-Teller distortion, which directly affects the electronic system by lifting the $e_{g}$ orbital degeneracy.60 Such distortions usually alternate between sites ("AF"), and experimentally they appear to influence the nature of the ground state primarily at low hole doping. However, opservation of stripe-like structures in manganite systems 61 has not yet been related unambiguously to lattice distortions, and may lie in the competition of next-neighbor repulsion $V$ with anisotropic conduction-electron hopping $t$.

With regard to methodology, the Hartree-Fock approach is generally understood to be "crude but effective". It is capable of finding the relevant ground states of the system, and allows one to draw many qualitative conclusions about their possible nature and order. However, the approximations involved in the breaking of symmetries and neglect of quantum fluctuations are sufficiently strong that the parameter ranges where different features appear, i.e. the quantitative aspect, may not be trusted. In addition, with periodic BCs, the self-consistency pro- 


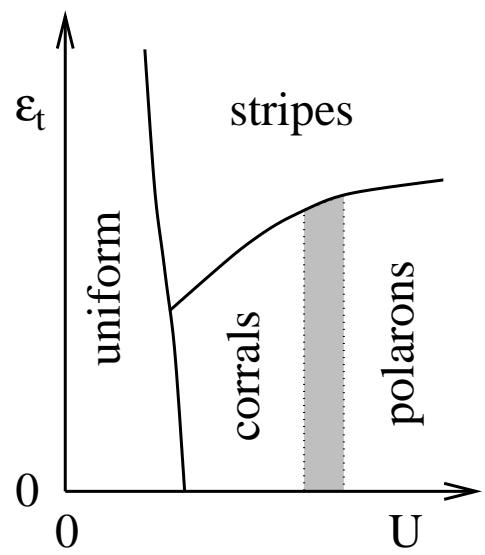

FIG. 16. Schematic phase diagram of HF solutions for the Hubbard model as a function of $U$ and hopping anisotropy $\epsilon_{t}$, for dopings $0.06<x<0.2$. The "uniform" region is a paramagnetic metal. The nature of the stripe phases depends on $x$, as discussed in Sec. IV. Solid lines are true phase transitions, while the character of charge-inhomogeneous solutions at large $U$ and small $\epsilon_{t}$ changes continuously.

cedure has difficulties converging adequately due to the large choice of (translationally) degenerate ground states. A possible solution to both problems js affered by the Configuration Interaction (CI) method.62 64 In this approach, the ground state is constructed as a (biased) linear combination of $\mathrm{HF}$ solutions. This technique, which has already been applied to certain aspects of the isotropic Hubbard model,63.51 can be shown to give a systematic description of quantum fluctuation and tunneling corrections to the HF solution, and of the quantum dynamics of the excitations.

While CI represents a significant improvement to HF, there remain many other techniques which could be applied to address the question of anisotropy effects at a more quantitative level. As listed in Sec. I, these include effective models of domain walls, the slave-boson representation, DMFT and DMRG, and we await with interest the results of subsequent analyses. We note finally that similar or adapted HF techniques may also be expanded to explore situations beyond the Hubbard and $t$ - $J$ models. In the framework of more general $t-J-U_{-} V$ models one-may seek finite, nearest-neighbor pairing 88 and spinflip 64 order parameters, with a view to discussing superconductivity and flux phases in charge and spin. The latter possibilities have been of considerable recent interest in both single- and multi-band models.

In conclusion, certain cuprate systems show a breaking of 4-fold $(x, y)$ symmetry in the $\mathrm{CuO}_{2}$ plane which is closely connected to the appearance of static stripes. Proceeding from this experimental motivation we have considered Hubbard and $t-J$ models with hopping and superexchange anisotropy. The real-space Hartree-Fock approach offers many possibilities by which to gain insight into the nature of the solutions. In the isotropic Hubbard model we find predominantly closed, diagonal domain-wall loops (corrals), and polarons at larger $U$. Anisotropy quite generally brings a gain in electronic energy, and introduces vertical stripes, which are stabilized by hopping between different charge densities. The qualitative situation is summarized in Fig. 16, which without distinguishing between types of stripe is applicable for the doping range of physical interest. The dominant, uniformly "filled" stripes are oriented perpendicular to the direction of strong hopping, have antiphase nature, and show in their ground-state energy the very strong influence of transverse hopping. For certain hole densities a non-uniform stripe appears, which may be the HF analogue of the "half-filled" stripes seen in experiment, and has longitudinal orientation and in-phase character. The isotropic $t$ - $J$ model in the HF approximation favors polaron formation and shows a tendency to phase separation with increasing $J$. Anisotropies in $t$ favor transverse stripe formation, while anisotropies in $J$ reinforce longitudinal orientation of stable stripes, but also act to promote phase separation. We conclude that stripes cannot be considered in isolation from the question of latticeinduced anisotropies.

\section{ACKNOWLEDGEMENTS}

We are grateful to C. Morais Smith, A. M. Oleś, T. M. Rice, D. J. Scalapino, M. Vojta, R. Werner, and S. R. White for invaluable discussions. This work was supported by the Deutsche Forschungsgemeinschaft through SFB 484.

${ }^{1}$ J. M. Tranquada, B. J. Sternlieb, J. D. Axe, Y. Nakamura, and S. Uchida, Nature (London) 375, 561 (1995).

2 J. M. Tranquada, J. D. Axe, N. Ichikawa, Y. Nakamura, S. Uchida, and B. Nachumi, Phys. Rev. Lett. 54, 7489 (1996).

${ }^{3}$ J. M. Tranquada, J. D. Axe, N. Ichikawa, A. R. Moodenbaugh, Y. Nakamura, and S. Uchida, Phys. Rev. Lett. 78, 338 (1997).

${ }^{4}$ K. Yamada, C. H. Lee, Y. Endoh, G. Shirane, R. J. Birgeneau, and M. A. Kastner, Physica C 282-287, 85 (1997).

${ }^{5}$ S. Wakimoto, G. Shirane, Y. Endoh, K. Hirota, S. Ueki, K. Yamada, R. J. Birgeneau, M. A. Kastner, Y. S. Lee, P. M. Gehring, and S. H. Lee, Phys. Rev. B 60, 769 (1999).

${ }^{6}$ S. Wakimoto, R. J. Birgeneau, M. A. Kastner, Y. S. Lee, R. Erwin, P. M. Gehring, S. H. Lee, M. Fujita, K. Yamada, Y. Endoh, K. Hirota, and G. Shirane, Phys. Rev. B 61, 3699 (2000).

${ }^{7}$ V. Emery, S. Kivelson, and O. Zachar, Phys. Rev. B 56, 6120 (1997). S. Kivelson, E. Fradkin, and V. Emery, Nature 375, 550 (1998).

${ }^{8}$ B. Büchner, M. Breuer, A. Freimuth, and A. Kampf, Phys. Rev. Lett. 73, 1841 (1994). 
${ }^{9}$ K. Levin, J. H. Kim, J. P. Lu, and Q. Si, Physica C 175, 449 (1991).

${ }^{10}$ P. B. Littlewood, J. Zaanen, G. Aeppli, and H. Monien, Phys. Rev. B 48, 487 (1994).

11 T. E. Mason, G. Aeppli, and H. A. Mook, Phys. Rev. Lett. 65, 2466 (1990).

12 S.-W. Cheong, G. Aeppli, T. E. Mason, H. A. Mook, S. M. Hayden, P. C. Canfield, Z. Fisk, K. N. Clause, and J. L. Martinez, Phys. Rev. Lett. 67, 1761 (1991).

${ }^{13}$ H. A. Mook, P. Dai, S. M. Hayden, G. Aeppli, T. Perring, and F. Dogan, Nature 395, 580 (1998).

${ }^{14}$ H. A. Mook and F. Dogan, Nature 401, 145 (1999).

${ }^{15}$ A. W. Hunt, P. M. Singer, K. R. Thurber, and T. Imai, Phys. Rev. Lett. 82, 4300 (1999).

${ }^{16}$ P. M. Singer, A. W. Hunt, A. F. Cederström, and T. Imai, Phys. Rev. B 60, 15345 (1999).

${ }^{17}$ N. Ichikawa, S. Uchida, J. M. Tranquada, T. Niemöller, P. M. Gehring, S.-H. Lee, and J. R. Schneider, Phys. Rev. Lett. 85, 1738 (2000).

${ }^{18}$ C. Morais Smith, A. H. Castro Neto, and A. V. Balatsky, cond-mat/0012080 (unpublished).

19 J. Zaanen and O. Gunnarsson, Phys. Rev. B 40, 7391 (1989).

${ }^{20}$ D. Poilblanc and T. M. Rice, Phys. Rev. B 39, 9749 (1989).

${ }^{21}$ H. J. Schulz, Phys. Rev. Lett. 62, 1445 (1990).

${ }^{22}$ M. Kato, K. Machida, H. Nakanishi, and M. Fujita, J. Phys. Soc. Jpn. 59, 1047 (1990).

23 T. Giamarchi and C. Lhuillier, Phys. Rev. B 42, 10641 (1990).

24 J. A. Vergés, E. Louis, P. S. Lomdahl, F. Guinea, and A. R. Bishop, Phys. Rev. B 43, 6099 (1991).

${ }^{25}$ H. E. Viertiö and T. M. Rice, J. Phys. Condens. Matter 6, 7091 (1994).

${ }^{26}$ V. J. Emery and S. A. Kivelson, Physica C 209, 597 (1993), S. A. Kivelson and V. J. Emery, p. 619 in Strongly Correlated Electronic Materials: The Los Alamos Symposium 1993, edited by K. S. Bedell et al. (Addison Wesley, Redwood City, CA, 1994).

27 J. Zaanen and A. M. Oleś, Ann. Phys. (Leipzig) 5, 224 (1996).

${ }^{28}$ I. Martin, G. Ortiz, A. V. Balatsky, and A. R. Bishop, cond-mat/0003316 and 0009067 (unpublished).

29 D. Góra, K. Rościszewski, and A. M. Oleś, Phys. Rev. B 60, 7429 (1999).

${ }^{30}$ G. Seibold, C. Castellani, C. DiCastro, and M. Grilli, Phys. Rev. B 58, 13506 (1998).

${ }^{31}$ S. R. White and D. J. Scalapino, Phys. Rev. Lett. 80, 1272 (1998); 81, 3227 (1998).

${ }^{32}$ S. R. White and D. J. Scalapino, Phys. Rev. B 60, R753 (1999); 61, 6320 (2000).

${ }^{33}$ G. B. Martins, C, Gazza, J. C. Xavier, M. Feiguin, and E. Dagotto, Phys. Rev. Lett. 84, 5844 (2000).

${ }^{34}$ M. Fleck, A. I. Liechtenstein, E. Pavarini, and A. M. Oleś, Phys. Rev. Lett. 84, 4962 (2000).

${ }^{35}$ A. H. Castro Neto and D. Hone, Phys. Rev. Lett. 76, 2165 (1996).

${ }^{36}$ A. H. Castro Neto, Z. Phys. B 103, 185 (1997).

${ }^{37}$ M. G. Zacher, R. Eder, E. Arrigoni, and W. Hanke, Phys. Rev. Lett. 85, 2585 (2000).

${ }^{38}$ B. Dabrowski, Z. Wang, K. Rogacki, J. D. Jorgensen, R.
L. Hitterman, J. L. Wagner, B. A. Hunter, P. G. Radaelli, and D. G. Hinks, Phys. Rev. Lett. 76, 1348 (1996).

${ }^{39}$ V. Kataev, B. Rameev, A. Validov, B. Büchner, M. Hücker, and R. Borowski, Phys. Rev. B 58, R11876 (1998).

${ }^{40}$ G. Teitel'baum, B. Büchner, and H. de Gronckel, Phys. Rev. Lett. 84, 2949 (2000).

41 A. Bianconi, G, Bianconi, S. Caprara, D. DiCastro, H. Oyanagi, and N. L. Saini, J. Phys. Condens. Matter 12, 10655 (2000).

42 B. Büchner, M. Braden, M. Cramm, W. Schabiltz, W. Schnelle, O. Hoffels, W. Braunisch, R. Müller, G. Heger, and D. Wohlleben, Physica C 185-189, 903 (1991).

${ }^{43}$ M. K. Crawford, R. L. Harlow, E. M. McCarron III, W. E. Farneth, J. D. Axe, H. Chou, and Q. Huang, Phys. Rev. B 44, 7749 (1991).

${ }^{44}$ N. E. Bonesteel, T. M. Rice, and F. C. Zhang, Phys. Rev. Lett. 68, 2684 (1992).

${ }^{45}$ H. Eskes and J. H. Jefferson, Phys. Rev. B 48, 9788 (1993).

${ }^{46}$ W. A. Harrison, Electronic Structure and the Properties of Solids (Freeman, San Francisco, 1980).

${ }^{47}$ A. P. Kampf, D. J. Scalapino, and S. R. White, unpublished.

${ }^{48}$ O. Baberski, A. Lang, O. Maldonado, M. Hücker, B. Büchner, and A. Freimuth, Europhys. Lett. 44, 335 (1998).

${ }^{49}$ R. Werner, M. Hücker, and B. Büchner, Phys. Rev. B 62, 3704 (2000).

${ }^{50}$ E. Dagotto, Rev. Mod. Phys. 66, 763 (1994), and references therein.

${ }^{51}$ E. Louis, F. Guinea, M. P. Lopez Sancho, and J. A. Vergés, cond-mat/0007319 (unpublished).

52 Y. Nagaoka, Phys. Rev. 147, 392 (1966).

53 S. R. White and I. Affleck, cond-mat/0011098 (unpublished).

${ }^{54}$ W. Putikka, M. Luchini, and M. Ogata, Phys. Rev. Lett. 69, 2288 (1991).

${ }^{55}$ E. Dagotto and J. Riera, Phys. Rev. Lett. 69, 2288 (1991).

${ }^{56}$ P. Prelovsek and X. Zotos, Phys. Rev. B 47, 5984 (1993).

${ }^{57}$ For a study of the $t-U-J$ model on a two-leg ladder see S. Daul, D. J. Scalapino, and S. R. White, Phys. Rev. Lett. 84, 4188 (2000).

${ }^{58}$ V. Sachan, D. Buttrey, J. Tranquada, J. Lorenzo, and G. Shirane, Phys. Rev. B 51, 12742 (1995).

${ }^{59}$ H. Yoshizawa, T. Kakeshita, R. Kajimoto, T. Tanabe, T. Katsufuji, and Y. Tokura, Phys. Rev. B 61, R854 (2000).

${ }^{60}$ M. Capone, D. Feinberg, and M. Grilli, Eur. Phys. J. B 17, 103 (2000).

${ }^{61}$ S. Mori, S.-W. Cheong, and C. T. Chen, Nature 392, 473 (1998).

${ }^{62}$ P. Fulde, Electron Correlations in Molecules and Solids, Vol. 100 of Springer Series in Solid State Sciences (Springer Verlag, Berlin, 1991).

${ }^{63}$ E. Louis, F. Guinea, M. P. Lopez Sancho, and J. A. Vergés, Phys. Rev. B 59, 14005 (1999).

${ }^{64}$ M. Berciu and S. John, Phys. Rev. B 61, 10015 (2000); ibid. 16454 (2000). 